Portland State University

PDXScholar

1987

\title{
Numerical solutions of continuous wave beam in nonlinear media
}

Jeffrey Huang

Portland State University

Follow this and additional works at: https://pdxscholar.library.pdx.edu/open_access_etds

Part of the Electrical and Computer Engineering Commons Let us know how access to this document benefits you.

Recommended Citation

Huang, Jeffrey, "Numerical solutions of continuous wave beam in nonlinear media" (1987). Dissertations and Theses. Paper 3742.

https://doi.org/10.15760/etd.5626

This Thesis is brought to you for free and open access. It has been accepted for inclusion in Dissertations and Theses by an authorized administrator of PDXScholar. Please contact us if we can make this document more accessible: pdxscholar@pdx.edu. 
AN ABSTRACT OF THE THESIS OF Jeffrey Huang for the Master of Science in Electrical Engineering presented July 23, 1987.

Title: NUMERICAL SOLUTIONS OF CONTINUOUS WAVE BEAM IN NONLINEAR MEDIA

APPROVED BY MEMBERS OF THE THESIS COMMITTEE:

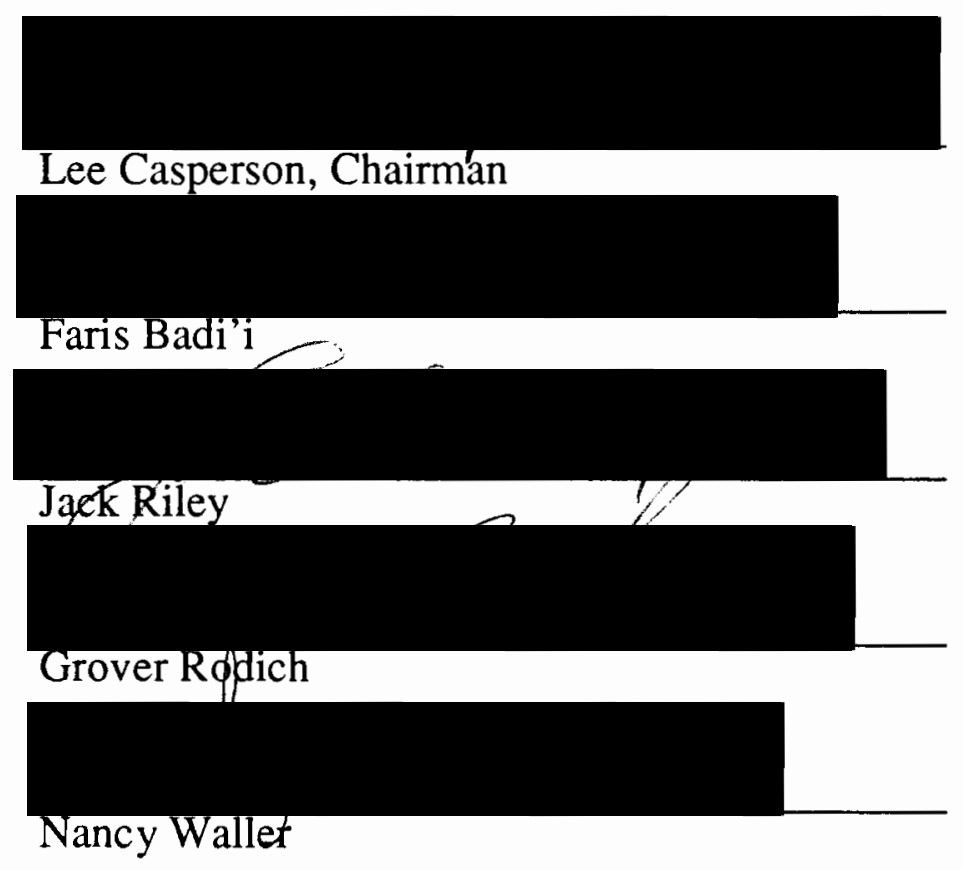

Deformation of a Gaussian beam is observed when it propagates through a plasma. Self-focusing of the beam may be observed when the intensity of the laser increases the index of refraction of plasma gas.

Due to the difficulties in solving the nonlinear partial differential equation in Maxwell's wave equation, a numerical technique has been developed in favor of the traditional analytical method. Result of numerical solution shows 
consistency with the analytical method. This further suggests the validity of the numerical technique employed.

A three dimensional graphics package was used to depict the numerical data obtained from the calculation. Plots from the data further show the deformation of the Gaussian beam as it propagates through the plasma gas. 
NUMERICAL SOLUTIONS OF CONTINUOUS WAVE BEAM IN NONLINEAR MEDIA

\author{
by \\ JEFFREY HUANG
}

A thesis submitted in partial fulfillment of the requirements for the degree of

\author{
MASTER OF SCIENCE \\ in \\ ELECTRICAL ENGINEERING
}

Portland State University 1987 
TO THE OFFICE OF GRADUATE STUDIES AND RESEARCH:

The members of the Committee approve the thesis of Jeffrey Huang presented July 23, 1987.

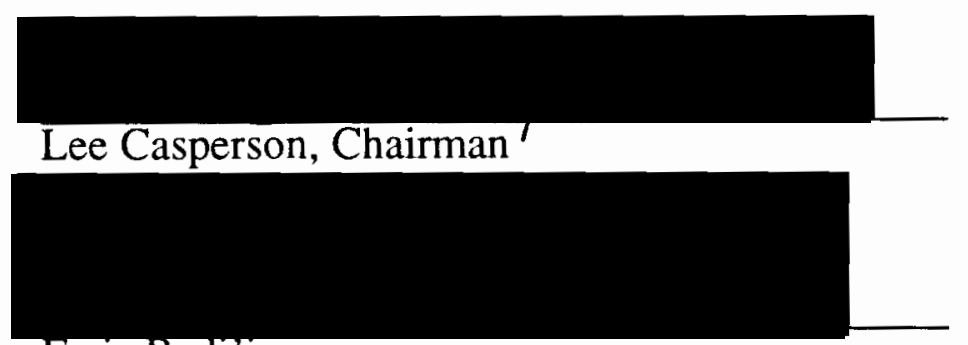

Faris Badr' 1

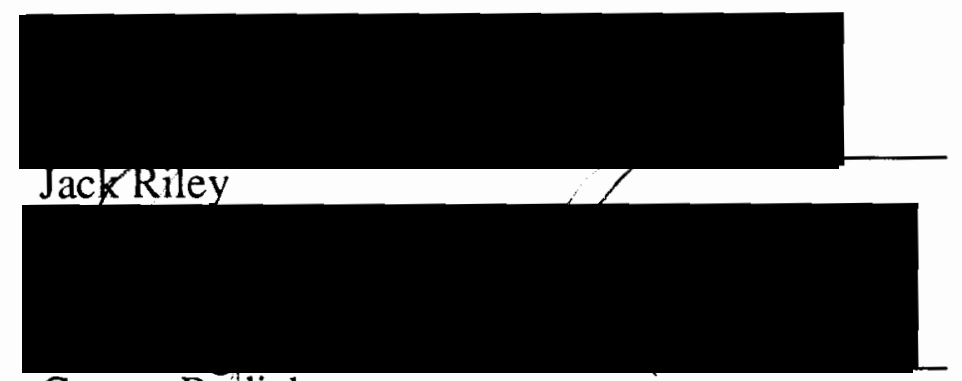

Grover Rodich

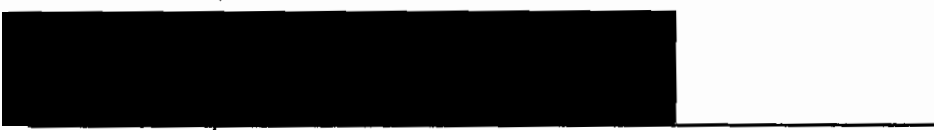

Nancy Wáller

APPROVED:

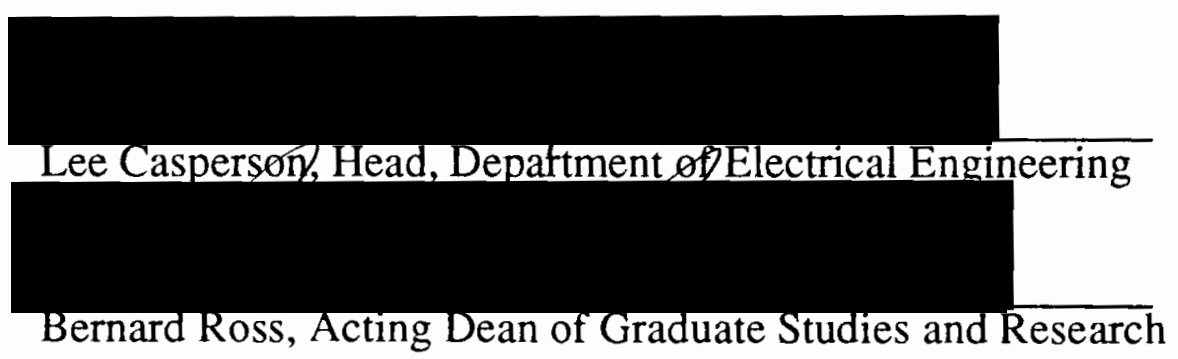




\section{ACKNOWLEDGEMENT}

"No man is an island", is what Mrs. Twinkle told me when I was in grade school. Although I never did get to know the works of John Donne, that phrase still rings clearly in my ears today. In retrospect, this paper would not have been possible if the following people had not made their individual contributions on my behalf. Because of these people, my life became fuller and changed forever.

I wish to extend my warmest appreciations:

To my parents, Sidney and Maria Huang; and my brothers, Jensen and Jimmy for all of their love and support.

To Dr. Lee Casperson for his encouragement, guidance and suggestions, and for allowing me the opportunity to be creative.

To Dr. Nancy Waller, who spent countless hours with me during the infancy of this thesis. Her endless patience and her command of the subject matter helped me tremendously with my task.

To Shirley Clark for her support and for proof-reading this paper.

To Janaka Jayawardena, for writing a special graphics package for me so that I could transmit graphs to the laser printer, and for making himself available to me whenever I needed his assistance.

To Adrian Fall, Rick Matt, Kapila Ranasinghe, and Tom Kautzman for their friendship.

J. H. 


\section{TABLE OF CONTENTS}

ACKNOWLEDGEMENT .......................................................................... ii

LIST OF TABLES ................................................................................... vi

LIST OF FIGURES ………........................................................................... vii

CHAPTER

I INTRODUCTION .......................................................................

II CONCEPTS OF DIFFERENTIAL EQUATIONS ........................ 5

Classification of Partial Differential Equations ....................... $\quad 7$

Initial and Boundary Conditions ............................................. 8

Nonlinearity ............................................................................... 9

Finite Difference Method ........................................................ 11

Taylor Series .......................................................................... 13

Variable Coefficient .................................................................. 18

III DERIVATION OF BEAM EQUATION .................................... 20

Maxwell's Equations .............................................................. 20

Numerical Solution ................................................................. 30

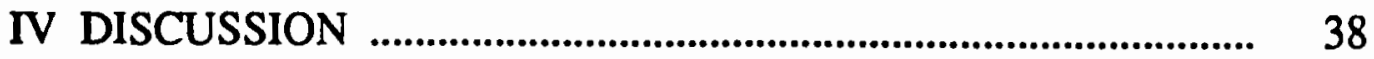


V CONCLUSION 46

BIBLIOGRAPHY 48

APPENDICES 50

I MAIN PROGRAM 51

II SUBROUTINES 63 III DEF FILES 67 


\section{LIST OF TABLES}

1. Finite difference approximations with one independent variable.

2. Finite difference approximations with two independent

variables. 


\section{LIST OF FIGURES}

2.1. Simple pendulum.

2.2. Two dimensional representation of $\mathrm{U}$ versus $\mathrm{x}$ with even grid spacing.

2.3 Two dimensional case utilitizing five points scheme.

2.4 Three dimensional case utilitizing seven points scheme.

3.1 Variation of field at $\mathrm{z}=0$.

3.2 Nodal points calculation for $\mathbf{z}=\mathbf{0}$ (on axis).

3.3 Nodal points calculation for $r>0, z>0$.

3.4 Nodal points calculation for $\mathrm{r}=0, \mathrm{z}>0$.

3.5 Nodal points calculation for $r>0, z>0$.

4.1 Cross-section of gaussian beam at the interface of the plasma cylinder.

4.2 Matrix mapping on the plasma cylinder.

4.3 Beam intensity profile in vacuum.

4.4 Beam intensity profile in plasma.

4.5(a) Steady state oscillations.

4.5(b) Steady state oscillations with rotation on intensity axis. 


\section{CHAPTER I}

\section{INTRODUCTION}

When man tries to understand the behavior of mother nature, he, if a scientist, would use the concepts of mathematics and physics to mimic that behavior by creating a mathematical model. These mathematical models often consist of one or more differential equations. This is particularly evident when one is studying the concept of motion. The motion of a particle consists of the trajectory or path this particle must take to get from point $A$ to point $B$. It also requires force, which is a phenomenon that drives the particle. This is also true when one studies the phenomena of heat diffusion, fluid flow, wave propagation, etc. The difference here is that we are dealing with partial differential equations. ${ }^{11}$

Once a mathematical model of interest is constructed, the ultimate task for the mathematician is to solve the differential equations or partial differential equations contained in that model. Unfortunately, there may not always be an analytical solution available. To circumvent these "minor" technicalities, one could become rather creative and perhaps ingeniously throw away terms in the mathematical model. After that, one might pat oneself on the back and claim that one had solved the differential equation at hand. This is done in the name of "simplifying the problem". The truth of the matter is that he has reduced a complex problem to a simpler model by disregarding terms which felt not to be 
significant. This may work in a classroom situation, but may not apply in the "real world" situation.

Since the advent of computers, some mathematicians and physicists have become bold and decided to get rid of the analytic solutions by trying to "squeeze" out as much information as they can out of the differential equation directly. " To do so, one may take the differential equation at hand, and transform it into a difference equation. Unfortunately this method is limited to a certain degree of accuracy. After all, the finite difference method is merely an approximation of the actual differential equation, although some purists may argue that the finite difference method is an exact duplicate of a differential equation. One must realize that a computational tool such as a computer has a limited amount of data storage space. Hence, truncation error is inevitable. One could, however, argue also that by reducing the step size, one could then reduce the errors that might accumulate. Nevertheless, there is still an error.

In spite of the inherent errors in the finite difference method, it is still a very attractive means of solving differential equations. As we have previously stated, the actual analytic solutions available for a given set of differential equations are few and far between. In some instances, there may not be a solution at all. This is especially true in nonlinear partial differential equations. Thus a numerical technique would seem practical in these types of situations.

In light of all these, the finite difference method is still in a rather "crude" state. ${ }^{11}$ Perhaps with the advances in computer architecture, and with the invention of a faster processor, the mathematical community will be enticed to break new ground in numerical analysis.

Realizations of nonlinear phenomena and theories became a reality with the advent of high-power lasers. The self-focusing phenomenon of a laser beam in a 
nonlinear medium became a special interest to many because of its influence on all other nonlinear phenomena ${ }^{9}$ Self-focusing phenomena within plasmas have generated a great deal of attention on account of their applicability to controlled thermonuclear fusion. Some have contemplated using laser beams to heat the plasma contained within reactors, since by doing so, one might relax the overall input power required in heating the reactors. ${ }^{6}$

The theory is to use the nonlinear characteristic of the plasma to enhance the intensity of the beam resulting from self-focusing. This in turn promotes the collisionless heating of the plasma. At large time scales ( $\geq \tau_{E}$, where $\tau_{E}$ is the energy relaxation time), the self-focusing of the beam gives rise to a large enhancement in the collisional (ohmic) heating of the plasma, which is again useful for fusion. The collisional heating of the plasma is also applicable to the heating of the ionosphere by a ground based HF transmitter, and has been of much interest in space communications and ionospheric diagnostics. ${ }^{9}$

Several mechanisms have been proposed for self-focusing in plasmas. The first of these is a basic consequence of the ponderomotive forces exerted on the plasma by the light beam. It would be useful to visualize physically the phenomenon of self-focusing in a medium where the refractive index is an increasing function of the intensity $\mathrm{EE}^{*}$ of the beam. ${ }^{9}$ In another words, the radiation pressure from the intense beam tends to drive electrons out of the beam resulting in a higher index of refraction at the center of the beam. Thus, a sufficiently intense electromagnetic wave can create its own waveguide. Another model for self-focusing postulates that the electron density near the beam center is "forced out" by the localized high temperature resulting from the beam. This mechanism has been proposed for obtaining beam confinement in the long laserheated thermonuclear reactors. 
In the present work, we are primarily interested in using numerical techniques to solve Maxwell's wave equation. Numerical techniques are favored over the traditional analytical methods, in part due to the difficulty in obtaining an explicit solution to the wave equation. Secondly, the traditional method requires simplification of the wave equation prior to solving it. This generally entails the linearization and disregard of terms that may seem difficult to integrate. As a consequence, the spirit behind the nonlinear partial differential equation has been lost forever.

In chapter two, we will review some of the fundamental concepts behind the finite difference method. I have make no attempt to cover everything, but deal only with those concepts that will be necessary for us to transform the analytical wave equation, which will be derived in chapter three, into the difference equation.

In chapter four, the results obtained from the numerical technique will be discussed. Except where noted, we will concentrate only on the characteristics of the laser beam in the plasma gas. The algorithm for this program utilizes the explicit form. It is the belief of the author that, for the present work, the explicit method is superior to the implicit method in the finite difference approximation. This is due to its greater simplicity in terms of coding and the consequently greater ease with which it may be altered in future. alteration which might arise. Although the implicit method is unconditionally stable for any chosen step size, the advantages forementioned still outweigh that of the implicit method. 


\section{CHAPTER II}

\section{CONCEPTS OF DIFFERENTIAL EQUATIONS}

Generally, notations used to denote independent variables have been $x, y, z$ and the dependent variables have been $u, v, w$. Thus $u, v$, and $w$ can be written as

$$
\begin{aligned}
& u=u(x, y, z) \\
& v=v(x, y, z) \\
& w=w(x, y, z)
\end{aligned}
$$

If one is interested in finding out the first derivative of $u$ as a function of $x$, one could differentiate $u$ and write it as follow

$$
u_{x}=\frac{\partial u}{\partial x}
$$

Similarly we can also write $v$ and $w$ with respect to $x$ as

$$
\begin{aligned}
& v_{x}=\frac{\partial v}{\partial x} \\
& w_{x}=\frac{\partial w}{\partial x}
\end{aligned}
$$

Now if we would like to take the partial derivative of $u_{x}, v_{x}$, and $w_{x}$, then we can further differentiate (2.2) and (2.3) and write them as

$$
\begin{aligned}
& u_{x y}=\frac{\partial^{2} u}{\partial y \partial x} \\
& v_{x y}=\frac{\partial^{2} v}{\partial y \partial x}
\end{aligned}
$$




$$
w_{x y}=\frac{\partial^{2} w}{\partial y \partial x}
$$

Understanding the above notation allows us to lump it all together, and claim that any partial differential equation (PDE) must have the following general form

$$
F\left(x, y, u, u_{x}, u_{y}, u_{x x}, u_{y y}, \dot{u}_{x y}, \ldots\right)=0
$$

where $\mathrm{F}$ is a function of the indicated quantities, with at least one argument being a differential equation.

In ordinary differential equations (ODE), the ordering can be determined rather easily. This is because in ODE we are dealing only with one independent and one dependent variable. However, in a PDE the ordering may not be so trivial. For example, a first order equation would look something like

$$
u_{x}+b y=0
$$

While a second order equation may look like

$$
u_{x x}=u_{y y}
$$

for an equation which contains several inter-related independent variables, we must reduce it to an equation involving only one independent variable before determining the order. Consider the following:

$$
u_{x}+v_{y}=u_{z}
$$

We will use substitution of variables by letting

$$
w_{x}=u
$$

and

$$
w_{y}=v
$$

Taking the first derivative of (2.9) with respect to $\mathrm{x}$ and (2.10) with respect to $\mathrm{y}$, 
we get

$$
w_{x x}=u_{x}
$$

and

$$
w_{y y}=v_{y}
$$

We also need to differentiate the righthand side of (2.8) with respect to $z$, which is equivalent to differentiating equation (2.9) with respect to $z$. This yields

$$
w_{x z}=u_{x}
$$

Combining equation (2.11) through (2.13), we get

$$
w_{x x}+w_{y y}=w_{x z}
$$

So if (2.8) was written in the form of (2.14), then it is apparent that (2.8) is of second order.

\section{CLASSIFICATION OF PARTIAL DIFFERENTIAL EQUATIONS}

The most general second order PDE in two independent variables has the form

$$
\begin{aligned}
& A(x, y) u_{x x}+2 B(x, y) u_{x y}+C(x, y) u_{y y}+D(x, y) u_{x} \\
& +E(x, y) u_{y}+F(x, y) u+G(x, y)=0
\end{aligned}
$$

where $A, B, C, D, E, F$, and $G$ are independent variables, constants, or functions of $x, y$ only. Subscripts denote the partial with respect to $x, y$, or both. Some of the famous second order PDEs are:

$$
\begin{aligned}
& u_{x x}+u_{y y}=0 \\
& u_{x x}+u_{y y}=f(x, y)
\end{aligned}
$$

Laplace's equation

Poisson's equation 


$$
\begin{array}{ll}
u_{x}=u_{r y} & \text { Heat flow or diffusion equation } \\
u_{x}=u_{y y}+u_{x z} & \text { Heat flow or diffusion equation } \\
k u_{y y}=u_{x}+u_{y} & \text { Burger's equation } \\
u_{x x}=u_{y y} & \text { Wave equation }
\end{array}
$$

A method has been devised to help categorize these PDEs into three canonical forms. Each form will suggest the type of boundary conditions required to solve the PDEs. Equation (2.15) can be categorized as hyperbolic, parabolic, or elliptic, if the following is satisfied:

$$
\begin{aligned}
& B^{2}-4 A C>0 \\
& B^{2}-4 A C=0 \\
& B^{2}-4 A C<0
\end{aligned}
$$

Hyperbolic

Parabolic

Elliptic

Thus the wave equation is hyperbolic since $A=1, B=0, C=-1$, so that

$$
B^{2}-4 A C=4>0
$$

\section{INITIAL AND BOUNDARY CONDITIONS}

In order to solve any PDE, it is important to establish some sort of initial and boundary conditions. After all, it is the initial conditions that determine a solution uniquely.

A solution of a partial differential equation is a function that satisfies the equation in some region of the space of the independent variables. In applications, we are generally less concerned with finding a general solution than with finding solutions satisfying the initial conditions or boundary conditions. ${ }^{13}$ The 
initial conditions concern values or derivatives of the solution, say at $t=0$, where $t$ is one of the independent variables (usually time), and we seek a solution for $t$ $=0$. The boundary conditions concern the values or derivatives of the unknown function on the boundary of the region (or interval) of the independent space variables. We often consider initial conditions as a form of boundary conditions and refer to a PDE with all the conditions as a boundary - value problem.

\section{NONLINEARITY}

What is nonlinearity? What significance does it have? Recall the most common phrase that has been mentioned time after time, "assume". For example, "assuming a homogeneous and isotropic medium, the equation becomes ...", "assume the fluid flow is inviscid,", or "assume a lossless medium ...". Naturally, we have been conditioned that everything is ideal, frictionless, incompressible, rigid, etc. ${ }^{12}$

Techniques for solving linear equations have been studied far more extensively than their counterparts - nonlinear equations. This is, in part, due to the complexity involved in solving nonlinear equations. Also, it is due to the lack of theory behind nonlinear equations. However, we are fortunate in the sense that "most" physical problems lead to a linear approximation. ${ }^{3}$ For instance, in the case of a simple oscillating pendulum problem, often cited in the elementary physics class: 


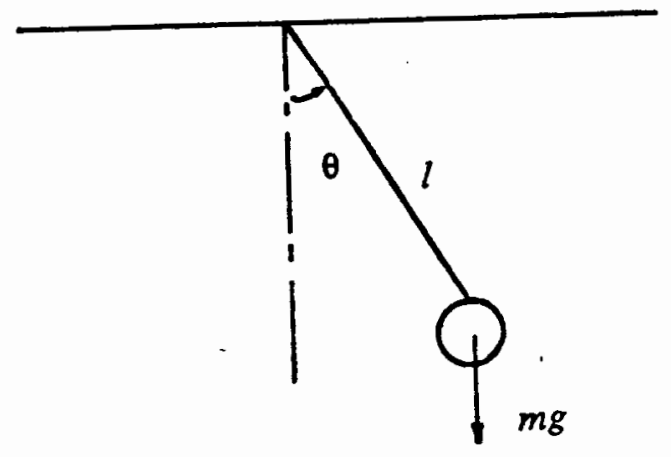

Figure 2.1. Simple pendulum.

The pendulum with a mass $m$ and attached string with length $l$ satisfies the nonlinear equation:

$$
\frac{d^{2} \theta}{d t^{2}}+\frac{g}{l} \sin (\theta)=0
$$

We can simplify the problem, by utilizing the small angle approximation, such that

$$
\sin (\theta)-\theta
$$

By doing so, we have linearized an otherwise nonlinear equation. This technique may suffice for some problems, but not for others. The solution obtained may or may not shed light on the behavior of the system being studied. It is also important to note that, if the equation is nonlinear, then with few exceptions, the solution to the equation may not be obtained explicitly. In this case, we can implement a numerical scheme to arrive at an acceptable solution without linearization.

It can be easily shown from the fundamentals of calculus, that the derivative of a sum of two functions equals the sum of the derivatives of the functions, such 
that

$$
\frac{d}{d x}(u \pm v)=\frac{d u}{d x} \pm \frac{d v}{d x}
$$

So we say, in general, if an operator is linear, then

$$
L(a u \pm b v)=a L u \pm b L v
$$

L can be a differential operator, $D$, or a constant, $\beta$, such that,

$$
\begin{aligned}
& D(a u \pm b v)=a D u \pm b D v \\
& \beta(a u \pm b v)=a \beta u \pm b \beta v
\end{aligned}
$$

\section{FINITE DIFFERENCE METHOD}

The finite difference method is a discrete technique, where the domain of interest is represented by a set of points or nodes as shown in figure (2.2). The method utilizes the mesh point technique where a net or mesh is thrown over the region of interest.

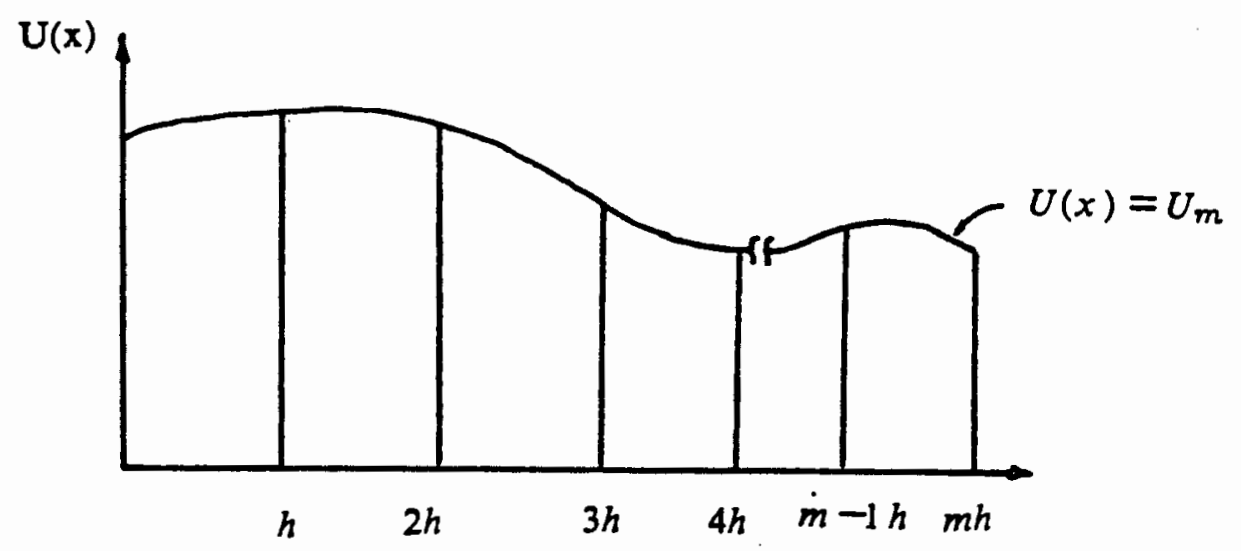

Figure 2.2. Two dimensional representation of some function of $\mathrm{U}$ vs $\mathrm{x}$ with even grid spacing. 
Now if $U(x)$ is a continuous function of the independent variable $x$, then we could discretize the $x$ domain by a set of points, $x_{n}$, such that

$$
\left.U(x)\right|_{x=x_{m}}=U\left(x_{m}\right)=U_{m}
$$

where

$$
\mathrm{m}=0,1,2, \ldots
$$

The grid spacing is defined by $h$, where $h$ is a constant.

For a two dimensional case, figure (2.3), the function $U(x, y)$ can be defined by $U\left(x_{m}, y_{n}\right)$.


Figure 2.3. Two dimensional case utilizing the five points scheme.

We can write a similar equation, and incorporate the nodal location as

$$
\left.U(x, y)\right|_{x=x_{n}, y=y_{0}}=U\left(x_{m}, y_{n}\right)=U_{m, n}
$$

where

$$
\begin{aligned}
& \mathrm{m}=0,1,2, \ldots . \\
& \mathrm{n}=0,1,2, \ldots .
\end{aligned}
$$

We can go one step further and expand the concept of two dimensional difference method to a three dimensional case, shown in figure (2.4). 

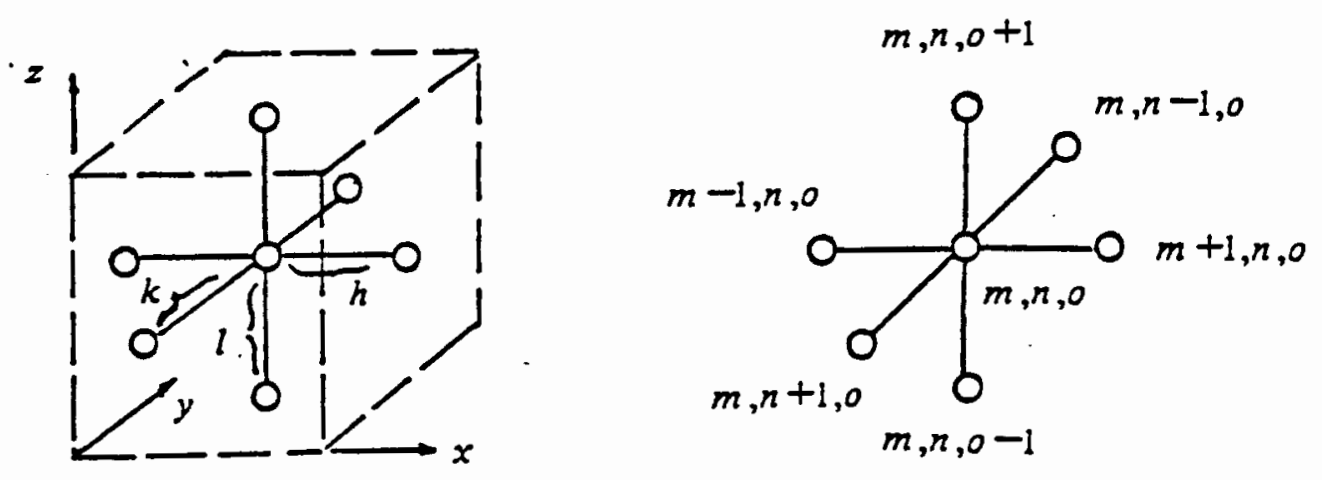

Figure 2.4. Three dimensional case using seven points scheme.

As before, we can write the three dimensional equation in terms of nodal location

$$
\left.U(x, y)\right|_{x=x_{,}, y=y_{,}, x=x_{0}}=U\left(x_{m}, y_{n}, z_{0}\right)=U_{m, n, 0}
$$

where

$$
\mathrm{m}, \mathrm{n}, \mathrm{o}=0,1,2, \ldots
$$

\section{TAYLOR SERIES}

The Taylor series plays an important role in the formulation of the finite difference method. Although there are many ways of obtaining the finite difference scheme, we shall focus only on the Taylor series, since the concept of other techniques are similar. ${ }^{1,11}$ For the one dimensional case, the incremental form is

$$
f\left(x_{n}+h\right)=f\left(x_{n}\right)+h f^{\prime}\left(x_{n}\right)+\frac{h^{2}}{2 !} f^{\prime \prime}\left(x_{n}\right)+\frac{h^{3}}{3 !} f^{\prime \prime}\left(x_{n}\right)+\cdots
$$

where $h$ is the step size in the $x$ direction and $x_{n}=n h$. 
Similarly, we can write the above equation as

$$
f\left(x_{n}-h\right)=f\left(x_{n}\right)-h f^{\prime}\left(x_{n}\right)+\frac{h^{2}}{2 !} f^{\prime \prime}\left(x_{n}\right)-\frac{h^{3}}{3 !} f^{\prime \prime}\left(x_{n}\right)+\cdots
$$

Solving for $f^{\prime}\left(x_{n}\right)$ from equation (2.22) we get

$$
f^{\prime}\left(x_{n}\right)=\frac{f\left(x_{n}+h\right)-f\left(x_{n}\right)}{h}-\frac{h}{2} f^{\prime \prime}\left(x_{n}\right)-\frac{h^{2}}{3 !} f^{\prime \prime \prime}\left(x_{n}\right)-\cdots
$$

or

$$
f^{\prime}\left(x_{n}\right)=\frac{f\left(x_{n}\right)-f\left(x_{n}+h\right)}{h}+\frac{h}{2} f^{\prime \prime}\left(x_{n}\right)-\frac{h^{2}}{3 !} f^{\prime \prime \prime}\left(x_{n}\right)-\cdots
$$

If we neglect the higher order terms,

$$
f^{\prime}\left(x_{n}\right)-\frac{f\left(x_{n}+h\right)-f\left(x_{n}\right)}{h}
$$

or

$$
f^{\prime}\left(x_{n}\right) \sim \frac{f\left(x_{n}\right)-f\left(x_{n}-h\right)}{h}
$$

Since the higher order terms have been neglected, we have introduced an error into our numerical solution. To determine the error, we will make use of a theorem in calculus which states that the total error is less than the maximum value of the first truncated term, when this term is evaluated over the interval ( $\left.x_{n}, x_{n}+h\right)$ or $\left(x_{n}, x_{n}-h\right)$. Thus,

$$
E_{n}= \pm \frac{h}{2 !} f^{\prime \prime}(\zeta)=O(h)
$$

where

$$
\begin{array}{ll}
x_{n} \leq \zeta \leq x_{n}+h & \text { (when }+ \text { is used) } \\
x_{n}-h \leq \zeta \leq x_{n} & \text { (when - is used) }
\end{array}
$$


We will denote $O(h)$ as the order of error, while $h$ determines the step size error. Naturally, the higher the order of $h$, the smaller the error. Combining equation (2.24) and (2.25) yields,

$$
f^{\prime}\left(x_{n}\right)=\frac{f\left(x_{n}+h\right)-f\left(x_{n}-h\right)}{2 h}-\frac{h^{2}}{3 !} f^{\prime \prime \prime}\left(x_{n}\right)-\cdots
$$

Neglecting the third derivative, the above equation reduces to

$$
f^{\prime}\left(x_{n}\right) \sim \frac{f\left(x_{n}+h\right)-f\left(x_{n}-h\right)}{2 h}
$$

with

$$
\begin{aligned}
& E_{n}=-\frac{h^{2}}{6 !} f^{\prime \prime \prime}(\zeta) \\
& x_{n}-h \leq \zeta \leq x_{n}+h
\end{aligned}
$$

Equation (2.30) is of $O\left(h^{2}\right)$. Now if we are interested in obtaining the second derivative we can simply subtract equations (2.24) and (2.25). This yields,

$$
f^{\prime \prime}\left(x_{n}\right)-\frac{f\left(x_{n}+h\right)-2 f\left(x_{n}\right)+f\left(x_{n}-h\right)}{h^{2}}
$$

with

$$
\begin{aligned}
& E_{n}=-\frac{h^{2}}{12} f^{4}(\zeta) \\
& x_{n}-h \leq \zeta \leq x_{n}+h
\end{aligned}
$$

It should be apparent that (2.32) is of the order $O\left(h^{2}\right)$. A list of the most useful approximations have been tabulated in tables (1) and (2). Table (1) is for one independent variable, and table (2) is for two independent variables. ${ }^{1}$ 
Finite Difference

Derivative

$U_{x} I_{r}$

$$
\frac{U_{r+1}-U_{r}}{h}
$$

$$
\frac{U_{r}-U_{r-1}}{h}
$$

$$
\frac{U_{r+1}-U_{r-1}}{2 h}
$$

$$
\frac{-U_{r+2}+4 U_{r+1}-3 U_{r}}{2 h}
$$

$$
\frac{-U_{r+2}+8 U_{r+1}-8 U_{r-1}+U_{r-2}}{12 h}
$$

$U_{x a} l_{r}$

$$
\frac{U_{r+1}-2 U_{r}+U_{r-1}}{h^{2}}
$$

$$
\frac{-U_{r+2}+16 U_{r+1}-30 U_{r}+16 U_{r-1}-U_{r-2}}{12 h^{2}}
$$

$U_{x x} l_{r}$

$$
\frac{U_{r+2}-2 U_{r+1}+2 U_{r-1}-U_{r-2}}{2 h^{3}}
$$

$\left.U_{4 x}\right|_{r}$

$$
\frac{U_{r+2}-4 U_{r+1}+6 U_{r}-4 U_{r-1}+U_{r-2}}{h^{4}}
$$

Table 1. Finite difference approximations with one independent variable. 
$\left.U_{x}\right|_{r s}$

$$
\begin{aligned}
& \frac{U_{r+1, s}-U_{r, s}}{h} \\
& \frac{U_{r, s}-U_{r-1, s}}{h}
\end{aligned}
$$

$$
\frac{U_{r+1, s}-U_{r-1, s}}{2 h}
$$

$$
\frac{-U_{r+2, s}+4 U_{r+1, s}-3 U_{r, s}}{2 h}
$$

$$
\frac{U_{r+1, s+1}-U_{r-1, s+1}-U_{r+1, s-1}+U_{r-1, s-1}}{12 h}
$$

$\left.U_{\mathbf{x z}}\right|_{r, s}$

$$
\frac{U_{r+1, s}-2 U_{r s}+U_{r-1 s}}{h^{2}}
$$

$$
\frac{-U_{r+2, s}+16 U_{r+1, s}-30 U_{r, s}+16 U_{r-1, s}-U_{r-2, s}}{12 h^{2}}
$$

$U_{4 x} I_{r, 5}$

$$
\frac{U_{r+2, s}-4 U_{r+1, s}+6 U_{r, s}-4 U_{r-1, s}+U_{r-2 s}}{h^{4}}
$$

$U_{x y} l_{r, s}$

$$
\frac{U_{r+1, s+1}-U_{r+1, s-1}-U_{r-1, s+1}+U_{r-1, s-1}}{4 h^{2}}
$$

$U_{x x y y} \mid r, s$

$$
\begin{gathered}
\frac{1}{h^{4}}\left[U_{r+1, s+1}+U_{r-1, s+1}+U_{r+1, s-1}+U_{r-1, s-1}-2 U_{r+1, s}\right. \\
\left.-2 U_{r-1, s}-2 U_{r, s+1}-2 U_{r, s-1}+4 U_{r, s}\right]
\end{gathered}
$$

Table 2. Finite difference approximations with two independent variables. 


\section{VARIABLE COEFFICIENT}

Many physical problems are described by PDE with variable coefficients, i.e., functions of $x, y$, etc. This is particularly evident in cylindrical and spherical coordinate systems. A wave equation, for instance, has the form

$$
\begin{array}{ll}
U_{x}=U_{y}+\frac{1}{y} U_{y} & \text { Cylindrical coordinate } \\
U_{x}=U_{y y}+\frac{2}{y} U_{y} & \text { Spherical coordinate }
\end{array}
$$

The above equations are similar, except for the constant, so we will write them in the general form

$$
U_{x}=U_{y y}+\frac{a}{y} U_{y}
$$

where $\quad a=0$ denotes Cartesian Coordinate

$a=1$ denotes Cylindrical Coordinate

$a=2$ denotes Spherical Coordinate

When $a=0$, equation (2.35) reduces to

$$
U_{x}=U_{y y} \quad \text { General heat equation }
$$

and we need not be concerned about $U$ at $y=0$. However, this become a major problem at $y=0$ for equation (2.35) if $a$ is not equal to zero, since at that point the differential equation becomes undefined.

To make the problem apparent, we will write (2.35) in terms of the difference equation.

$$
\begin{gathered}
\frac{U_{m+1, n}-U_{m, n}}{h}=\frac{U_{m,+1}-2 U_{m, n}+U_{m, n-1}}{k^{2}} \\
+\frac{a}{y_{n}}\left[\frac{U_{m, n+1}-U_{m,-1}}{2 k}\right]
\end{gathered}
$$


Note that at $y_{n}=0, \frac{a}{y_{n}}$ is undefined. To circumvent this problem we will assume that $U_{y}=0$ at $n=0$. This will allow us to use L'Hospital's rule, which states

$$
\lim _{x \rightarrow 0} \frac{f(x)}{g(x)}=\lim _{x \rightarrow 0} \frac{f^{\prime}(x)}{g^{\prime}(x)}
$$

Applying this principle to equation (2.35), we find that

$$
U_{y y}+\lim _{y \rightarrow 0}\left[\frac{a}{y_{n}} U_{y}\right]=(1+a) U_{y y}
$$

We will show in chapter four that this assumption is valid. But the fact that $U_{y}=0$ at $y=0$ implies that

$$
U_{m, 1}=U_{m,-1}
$$

This is the result of

$$
U_{y} I_{m, 0}=\frac{U_{m, 1}-U_{m,-1}}{2 k}=0
$$

and consequently the equation (2.36) reduces down to

$$
\frac{U_{m+1,0}-U_{m, 0}}{h}=\frac{2(1+a)}{k^{2}}\left(U_{m, 1}-U_{m, 0}\right)
$$

It has been shown by Lapidus and Pinder ${ }^{1}$ that equation (2.39) is conditionally stable within the region

$$
0<\rho \leq \frac{1}{2(1+a)}
$$

where $\rho=\frac{h}{k^{2}}$.

So for cartesian coordinates, $a=0, \rho \leq 1 / 2$. For cylindrical coordinate, $a=1$, the stability is limited to $\rho \leq 1 / 4$ and in spherical coordinates, $a=2, \rho \leq 1 / 6$. 
CHAPTER III

\section{DERIVATION OF BEAM EQUATION}

\section{MAXWELL'S EQUATION}

To understand how an electromagnetic wave propagates in a conducting media, it is very helpful to introduce the concept of effective dielectric constant from Maxwell's equations and constitutive relations, valid for conducting media. ${ }^{9}$ Maxwell's equations are

$$
\begin{aligned}
& \nabla \cdot \bar{D}=\rho \\
& \nabla \cdot \bar{B}=0 \\
& \nabla \times \bar{E}=-\frac{\partial \bar{B}}{\partial t}=-\mu \frac{\partial \bar{H}}{\partial t} \\
& \nabla \times \bar{H}=\bar{J}_{f}+\frac{\partial \bar{D}}{\partial t}
\end{aligned}
$$

where $\bar{E}$ and $\bar{D}$ are electric field and electric displacement vectors, $\vec{H}$ and $\bar{B}$ are the magnetic field and magnetic induction vectors, $\bar{J}_{f}$ is the free current density, $\rho$ is the free charge density, and $\varepsilon$ is the electric permittivity.

The constitutive relations in the conducting media are

$$
\bar{D}=\varepsilon \bar{E}
$$

and 


$$
\bar{B}=\mu \bar{H}
$$

Here $\mu$ is the magnetic permeability of the medium, and is approximately equal to unity for a plasma gas. ${ }^{4}$

From the continuity relationship, the charge density is related to the current density via

$$
\nabla \cdot \bar{J}+\frac{\partial \rho}{\partial t}=0
$$

The steady state current density is defined as

$$
\bar{J}=\sigma \bar{E}
$$

where $\sigma$ is the conductivity (complex in nature) of the plasma.

In a nonlinear inhomogeneous medium, the displacement vector may not be linear with $\bar{E}$, due to polarization. Thus we will need to add additional terms to equation (3.1e), such that

$$
\bar{D}=\varepsilon \bar{E}+\bar{P}_{L}+\bar{P}_{N L}
$$

where the subscripts $L$ and $N L$ denotes linear and nonlinear terms, respectively. Inserting (3.3) and (3.2b) into (3.1e) we get

$$
\nabla \times \bar{H}=\sigma \bar{E}+\frac{\partial}{\partial t}\left[\varepsilon_{0} \bar{E}+\bar{P}_{L}+\bar{P}_{N L}\right]
$$

If we were to take the left hand side of equation (3.4) and differentiate with respect to time, then the curl of $\bar{H}$ becomes

$$
\frac{\partial}{\partial t}(\nabla \times \bar{H})=\nabla \times \frac{\partial \bar{H}}{\partial t}=\frac{-1}{\mu}(\nabla \times \nabla \times \bar{E})
$$

From the vector identities' second derivative rule,

$$
\nabla \times \nabla \times A=\nabla \nabla \cdot A-\nabla^{2} A
$$


Then we can write (3.5a) as

$$
-\frac{1}{\mu} \nabla \times \nabla \times \bar{E}=\frac{-1}{\mu}\left(\nabla \nabla \cdot \bar{E}-\nabla^{2} \bar{E}\right)
$$

If we assume that there are no free electrons, $\rho=0$, and $\varepsilon$ is negligible in one wavelength, then

$$
\nabla \cdot \bar{E}=\frac{\rho}{\varepsilon}=0
$$

then $(3.5 b)$ reduces to

$$
-\frac{1}{\mu} \nabla \times \nabla \times \bar{E}=+\frac{1}{\mu} \nabla^{2} \bar{E}
$$

Replacing the right hand side of (3.4) with (3.5c), and differentiating the right hand side of (3.4)

$$
\begin{gathered}
\frac{1}{\mu} \nabla^{2} \bar{E}=\frac{\partial}{\partial t}\left[\sigma \bar{E}+\frac{\partial}{\partial t}\left[\varepsilon_{o} \bar{E}+\bar{P}_{L}+\bar{P}_{N L}\right]\right] \\
=\sigma \frac{\partial \bar{E}}{\partial t}+\varepsilon_{o} \frac{\partial^{2} \bar{E}}{\partial t}+\frac{\partial t^{2} \bar{P}_{L}}{\partial t^{2}}+\frac{\partial^{2} \bar{P}_{N L}}{\partial t^{2}}
\end{gathered}
$$

where

$$
\begin{aligned}
& \bar{P}_{L}=\varepsilon_{o} \chi_{2} \bar{E} \\
& \bar{P}_{N L}=\varepsilon_{o} \chi_{2}<\bar{E} \cdot \bar{E}>\bar{E} \\
& \varepsilon=\varepsilon_{o}\left(1+\chi_{e}\right)
\end{aligned}
$$

$<$ - > denotes time average of the field over many cycles. Rearranging (3.6b), we get the wave equation

$$
\nabla^{2} \bar{E}-\mu \varepsilon \frac{\partial^{2} \bar{E}}{\partial t^{2}}-\mu \sigma \frac{\partial \bar{E}}{\partial t}=\mu \frac{\partial^{2} \bar{P}_{N Z}}{\partial t^{2}}
$$

In the case of a field which is linearly polarized in the $x$ direction, equation (3.7a) 
can be written in the scalar form

$$
\nabla^{2} E_{x}-\mu \varepsilon \frac{\partial^{2} E_{x}}{\partial t^{2}}-\mu \sigma \frac{\partial E_{x}}{\partial t}=\mu \frac{\partial^{2} P_{N L_{x}}}{\partial t^{2}}
$$

For a harmonic time dependence, we will assume $E_{x}$ and $P_{x}$ to have the following forms,

$$
\begin{aligned}
& E_{x}=E^{\prime} \cos (\omega t) \\
& P_{x}=C \cos (\omega t)+i S \sin (\omega t)
\end{aligned}
$$

where $E_{x}$ is the force on the charge cloud and $P_{x}$ is the displacement of the charge cloud in the $x$ direction only. For simplicity, we will rewrite equation (3.8a) and (3.8b) in the phasor notation.

$$
\begin{aligned}
& E_{x}=\operatorname{Re}\left[E^{\prime} e^{i \omega x}\right] \\
& P_{x}=\operatorname{Re}\left[C e^{i \omega x}-i S e^{i \omega t}\right]
\end{aligned}
$$

Differentiating (3.9a) and (3.9b) twice with respect to time,

$$
\begin{aligned}
& \frac{\partial^{2} E_{x}}{\partial t^{2}}=-\omega^{2} E^{\prime} e^{i \omega t} \\
& \frac{\partial^{2} P_{x}}{\partial t^{2}}=-\omega^{2} C e^{i \alpha x}+i \omega^{2} S e^{i \omega x}
\end{aligned}
$$

Then the wave equation (3.7) has the following form,

$$
\nabla^{2} E^{\prime} e^{i \omega x}+\mu \varepsilon \omega^{2} E^{\prime} e^{i \omega x}-i \mu \sigma \omega E^{\prime} e^{i \omega x}=-\mu \omega^{2}\left[C e^{i \omega x}-i S e^{i \omega x}\right]
$$

Dividing (3.11) by $e^{i o t}$

$$
\nabla^{2} E^{\prime}+\mu \varepsilon \omega^{2} E^{\prime}-i \mu \sigma \omega E^{\prime}=-\mu \omega^{2}[C-i S]
$$

let 


$$
\begin{aligned}
& C=\varepsilon \chi^{\prime} E^{\prime} \\
& S=-\varepsilon \chi^{\prime \prime} E^{\prime}
\end{aligned}
$$

and expand (3.12)

$$
\nabla^{2} E^{\prime}+\mu \varepsilon \omega^{2} E^{\prime}-i \mu \sigma \omega E^{\prime}=-\mu \omega^{2} \varepsilon \chi^{\prime} E^{\prime}-i \mu \omega^{2} \varepsilon \chi^{\prime \prime} E^{\prime}
$$

It is important to point out that the transformation from (3.7) to (3.13) has allowed us to solve the wave equation at hand, since equation (3.7) consists of two unknowns in one equation, namely $\bar{P}_{N L}$ and $\bar{E}$. Grouping terms in (3.13),

$$
\nabla^{2} E^{\prime}+\left[\mu \varepsilon \omega^{2}\left(1+\chi^{\prime}\right)+i \mu \varepsilon \omega^{2}\left(\chi^{\prime \prime}-\frac{\sigma}{\varepsilon \omega}\right)\right] E^{\prime}=0
$$

If the spatial variation of $\varepsilon, \mu$ and $\sigma$ are negligible at a distance of one wavelength, it follows from (3.14) that the transverse cartesian components of the electric fields are governed by the simplified Helmholtz wave equation.

$$
\nabla^{2} E^{\prime}+K^{2} E^{\prime}=0
$$

where

$$
K^{2}=\mu \varepsilon \omega^{2}\left(1+\chi \dot{)}+i \mu \varepsilon \omega^{2}\left(\chi^{\prime \prime}-\frac{\sigma}{\varepsilon \omega}\right)\right.
$$

$K$ is defined as the wave number or propagation constant. Equation (3.16) also suggests that the propagation vector $K$ is complex, and it is designated by $\beta$ and $\alpha$ respectively. That is, $K=\beta+i \alpha$, where $\alpha$ is defined as the gain or loss term, and $\beta$ is the index term.

From the Drude model for a classical electron gas, ${ }^{10}$ the plasma conductivity is defined as

$$
\sigma_{p}=\frac{n e^{2}}{m\left(g+i \omega_{o}\right)}
$$


where

$$
\begin{aligned}
& \mathrm{m}=\text { electron mass } \\
& \mathrm{g}=\text { collision frequency } \\
& \mathrm{n}=\text { electron density } \\
& \mathrm{e}=\text { electron charge } \\
& \omega_{o}=\text { optical frequency }
\end{aligned}
$$

If we assume that the collision frequency is unimportant in our study, and susceptibility $\chi^{\prime}$ and $\chi^{\prime \prime}$ are negligible such that,

$$
\chi^{\prime}=\chi^{\prime \prime}=0
$$

Then combining (3.17) and (3.16) yields

$$
K^{2}=\mu \varepsilon \omega^{2}\left(1+i \frac{\omega_{p}^{2}}{\omega_{o}^{2}}\right)
$$

where $\omega_{p}=\sqrt{\frac{\widehat{n e^{2}}}{\varepsilon m}}$ is the electron plasma frequency. ${ }^{4}$ It should be apparent that equation (3.18) yields a solution of the complex form. Thus, we shall call equation (3.18) the complex index of refraction. ${ }^{4}$ The imaginary part of $K$ results in an attenuation of the wave (decreasing amplitude with increasing distance away from the origin). Since the propagation vector $K$ could also be written (without classifying the index of refraction as real or complex) as

$$
K=\frac{n \omega}{c}
$$

where

$$
\begin{aligned}
& n=\text { index of refraction } \\
& c=\text { speed of light } \\
& \omega=\text { frequency }
\end{aligned}
$$


Equating (3.19) and (3.18) and solving for the index of refraction

$$
n_{i}^{2}-1-i \frac{\omega_{p}^{2}}{\omega_{o}^{2}}
$$

For frequencies $\omega_{o}$ much less than $\omega_{p}, K$ is purely imaginary, and the waves attenuate away. ${ }^{10}$ For frequencies higher than $\omega_{p}$, on the other hand, the wave number is real, and the waves propagate without attenuation at a wave speed

$$
v=\frac{\omega}{K}=c\left[1-\left[\frac{\omega_{p}}{\omega_{0}}\right]^{2}\right]^{-1 / 2}
$$

Therefore, the plasma is opaque to waves of frequency less than $\omega_{p}$ and transparent to those above $\omega_{p}$.

The ponderomotive force acting on the plasma electrons in the presence of an electromagnetic field has an effect on the density of the plasma electrons. The plasma density ${ }^{6}$ is defined as

$$
n_{e}=n_{o e} e^{-\frac{\gamma|E|^{2}}{2}}
$$

where

$$
\begin{aligned}
& n_{e}=\text { electron density } \\
& n_{o e}=\text { electron density in the absence of } E-\text { field } \\
& y=\frac{e^{2}}{2 m \omega^{2} K\left(T_{e}+T_{i}\right)}
\end{aligned}
$$

Therefore, the plasma frequency ${ }^{6,10}$ is

$$
\omega_{p}^{2}=\omega_{p o}^{2} e^{-\frac{\gamma|E|^{2}}{2}}
$$

where $\omega_{p o}=$ plasma frequency in the absence of $E-$ field. 
Rewriting (3.14) in terms of plasma frequency

$$
\nabla^{2} E^{\prime}+\omega_{o}^{2} \mu \varepsilon\left[1-\frac{\omega_{p o}^{2}}{\omega_{o}^{2}} e^{-\frac{\gamma|E|^{2}}{2}}\right] E^{\prime}=0
$$

In cylindrical coordinates ( 3.23 ) could be written as

$$
\begin{aligned}
& \frac{\partial^{2} E^{\prime}}{\partial r^{2}}+\frac{1}{r} \frac{\partial E^{\prime}}{\partial r}+\frac{1}{r^{2}} \frac{\partial^{2} E^{\prime}}{\partial r^{2}}+\frac{\partial^{2} E^{\prime}}{\partial z^{2}} \\
& +\omega_{o}^{2} \mu \varepsilon\left[1-\frac{\omega_{p o}^{2}}{\omega_{o}^{2}} e^{-\frac{\gamma|E|^{2}}{2}}\right] E^{\prime}=0
\end{aligned}
$$

For a nearly plane wave propagating in the $\mathrm{z}$ direction

$$
E(r, \phi, z)=G(r, \phi, z) \exp \left[-i \omega(\mu \varepsilon)^{1 / 2}\left(1-\frac{\omega_{p o}^{2}}{\omega_{o}^{2}}\right)^{1 / 2} z\right]
$$

For an axial symmetrical beam, and assuming that $G$ varies so slowly with respect to $z$ that its second derivative can be neglected, (3.24) becomes

$$
\begin{gathered}
\frac{\partial^{2} G}{\partial r^{2}}+\frac{1}{r} \frac{\partial G}{\partial r}-i 2 \omega_{o}(\mu \varepsilon)^{1 / 2}\left[1-\frac{\omega_{p o}^{2}}{\omega_{o}^{2}}\right]^{1 / 2} \frac{\partial G}{\partial z} \\
+\omega_{p o}^{2} \mu \varepsilon\left[1-e^{-\frac{\left.|\gamma| G\right|^{2}}{2}}\right] G=0
\end{gathered}
$$

The general form for a Gaussian beam propagating in a uniform medium parallel to the $\mathrm{z}$ axis is

$$
G(r, z)=A(z) e^{-\frac{i Q(z) r^{2}}{2}}
$$

Equation (3.27) is also widely used as an approximation for a beam that is propagating in a nonuniform medium parallel to the $z$ axis. The complex 
parameter $Q(z)$ governs the spot size and the phase front curvature of the beam according to

$$
Q(z)=\frac{2 \pi}{\lambda R(z)}-i \frac{2}{w_{s}^{2}}
$$

The spot size, $w_{s}$, of the beam is determined by the amount of amplitude drop from its peak value at $r=0$ to $r=\frac{1}{e}$, figure (3.1), as the radius $r$ increases.

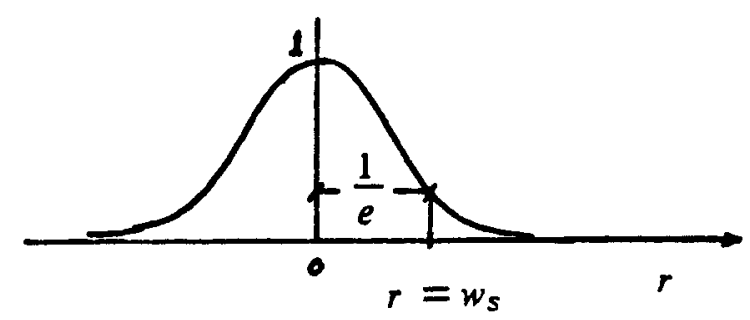

Figure 3.1. Variation of field at $\mathrm{z}=0$.

Since our eyes react to light as a function of intensity, we would observe that the major portion of the light lies within a small spot. This spot of light is known as the beam radius, otherwise as spot size $w_{s}$. For free space, the spot size is found to be

$$
w_{s}^{2}=w_{s 0}^{2}\left[1+\left[\frac{z}{z_{0}}\right]^{2}\right]
$$

and the radius of curvature $R(z)$ is

$$
R(z)=z\left[1+\left[\frac{z_{0}}{z}\right]^{2}\right]
$$

It should be intuitively apparent that if the phase front of the beam is not planar, then it is curved. At large $z, z \gg z_{0}$, the beam appears to be originating from $z=0$. As we approach the point $z=0$, the center of curvature goes to infinity. This is when the radius is planar. 
We can simplify (3.26) substantially by introducing normalized parameters for spot size $w_{s}^{\prime}$, phase front curvature $R^{\prime}(z)$, longitudinal distance $z^{\prime}$, radial distance $r^{\prime}$, plasma frequency $\alpha$, and power $P^{\prime}$ as follows:

$$
\begin{aligned}
& w_{s}^{\prime}(z)=\frac{\sqrt{2} \pi w_{s}(z)}{\lambda} \\
& R^{\prime}(z)=\frac{2 \pi R(z)}{\lambda} \\
& z^{\prime}=\frac{2 \pi z}{\lambda} \\
& r^{\prime}=\frac{\sqrt{2} \pi r}{\lambda} \\
& \alpha=\frac{\omega_{p o}^{2}}{\omega_{0}^{2}} \\
& P^{\prime}=\frac{4 \pi \gamma c \mu P}{\lambda^{2}} \\
& F=\sqrt{\frac{Y}{2}} G \\
& \lambda=\frac{2 \pi}{\omega_{o} \sqrt{\mu \varepsilon} \sqrt{1-\alpha}}
\end{aligned}
$$

Here we will introduce $\lambda$ as the wavelength of the medium. Now, insert the newly defined definition into (3.26) and we get

$$
\frac{\partial^{2} F}{\partial r^{\prime 2}}+\frac{1}{r^{\prime}} \frac{\partial F}{\partial r^{\prime}}-i 4 \frac{\partial F}{\partial z^{\prime}}+\frac{2 \alpha}{1-\alpha}\left[1-e^{-F^{2}}\right] F=0
$$

Aside from normalization, equation (3.31) is identical to that of (3.26). Thus, the integrity of the wave equation has been preserved. 


\section{NUMERICAL SOLUTION}

We are now in the position to transform the analytic equation (3.31) into the difference equation previously discussed.

$$
\begin{aligned}
& \frac{F(r+1, z)-2 F(r, z)+F(r-1, z)}{h^{2}}+\frac{1}{r_{s}} \frac{F(r+1, z)-F(r-1, z)}{2 h} \\
& -i 4\left[\frac{F(r, z+1)-F(r, z)}{k}\right]+\frac{2 \alpha}{(1-\alpha)}\left[1-e^{-F^{2}(r, z)}\right] F(r, z)=0
\end{aligned}
$$

But equation (3.31) contains a variable coefficient, namely

$$
\frac{1}{r_{s}} \frac{\partial F}{\partial r}
$$

So equation (3.32) will explode when $s=0$, or rather, at $r=0$. From our previous discussion, we can get around this by using L' Hospital's rule. Furthermore, we will need to split the equation into two parts; one for the "on axis" calculation, and the other for "off axis" calculation. Rewriting (3.31) with $a=1$ (cylindrical coordinate), we get

$$
2 \frac{\partial^{2} F}{\partial r^{\prime 2}}-i 4 \frac{\partial F}{\partial z^{\prime}}+\frac{2 \alpha}{(1-\alpha)}\left[1-e^{-F^{2}}\right] F=0
$$

Writing (3.33) in terms of $\frac{\partial F}{\partial z}{ }^{\prime}$

$$
\frac{\partial F}{\partial z^{\prime}}=-\frac{i}{2} \frac{\partial^{2} F}{\partial r^{\prime 2}}-\frac{i \alpha}{2(1-\alpha)}\left[1-e^{-F^{2}}\right] F
$$

In the numerical method representation, equation (3.34) becomes

$$
\begin{aligned}
& \frac{F(r, z+1)-F(r, z)}{k}=-\frac{i}{2}\left[\frac{F(r+1, z)-2 F(r, z)+F(r-1, z)}{h^{2}}\right] \\
& -\frac{i \alpha}{2(1-\alpha)}\left[1-e^{-F^{2}(r, z)}\right] F(r, z)
\end{aligned}
$$


Solving for $F(r, z+1)$ and grouping like terms

$$
\begin{aligned}
& F(r, z+1)=-\frac{i k}{2 h^{2}}[F(r+1, z)+F(r-1, z)] \\
& \quad+F(r, z)\left[1+\frac{i k}{h^{2}}-\frac{i k \alpha}{2(1-\alpha)}+\frac{i k \alpha}{2(1-\alpha)} e^{-F^{\prime}(r, z)}\right]
\end{aligned}
$$

Defining $\rho=\frac{k}{2 h^{2}}$, equation (3.36) can be written as

$$
\begin{aligned}
& F(r, z+1)=-i \rho[F(r+1, z)+F(r-1, z)]+ \\
& F(r, z)\left[1+i 2 \rho-\frac{i k \alpha}{2(1-\alpha)}+\frac{i k \alpha}{2(1-\alpha)} e^{r^{(t}(r, z)}\right]
\end{aligned}
$$

Recalling that symmetry exists for the on axis calculation, see figure (3.2).

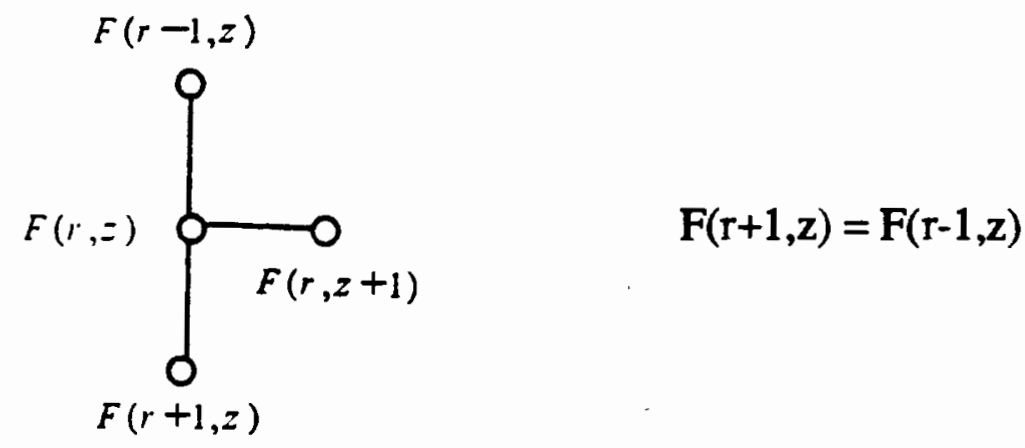

Figure 3.2. Nodal points calculation for $\mathrm{z}=0$ (on axis).

This permits us to reduce (3.37) further:

$$
\begin{aligned}
& F(r, z+1)=-i 2 \rho F(r+1, z) \\
& \quad+F(r, z)\left[1+i 2 \rho-\frac{i k \alpha}{2(1-\alpha)}+\frac{i k \alpha}{2(1-\alpha)} e^{-F(r, z)}\right]
\end{aligned}
$$

It is interesting how we could still obtain a solution when only two nodes were 
involved in solving for the third node. Thus it is important to recognize that stability may be non-existent if this method is applied throughout the entire calculation. It should also be apparent that the step size should be chosen to be small. The truncation error for (3.38) is

$$
E_{r}=O\left(k, h^{2}\right)
$$

$E_{r}$ will be large if $k$ is large compared to $h$, so care must be taken when selecting the step size, in both the $r$ and $z$ directions.

For the "off axis" calculation, solving for $\frac{\partial F}{\partial z}$ from (3.31)

$$
\frac{\partial F}{\partial z}=\frac{-i}{4} \frac{\partial^{2} F}{\partial r^{\prime 2}}-\frac{i}{4 r^{\prime}} \frac{\partial F}{\partial r^{\prime}}-\frac{i \alpha}{2(1-\alpha)}\left[1-e^{-F^{2}}\right] F
$$

Solving for $F(r, z+1)$ in the numerical method form,

$$
\begin{aligned}
& F(r, z+1)=F(r+1, z)\left[-\frac{i k}{4 h^{2}}-\frac{i k}{8 h r_{s}^{\prime}}\right] \\
& -F(r-1, z)\left[-\frac{i k}{4 h^{2}}+\frac{i k}{8 h r_{s}^{\prime}}\right] \\
& +F(r, z)\left[1+\frac{i k}{2 h^{2}}-\frac{i \alpha k}{2(1-\alpha)}+\frac{i \alpha k}{2(1-\alpha)} e^{-F^{2}(r, z)}\right]
\end{aligned}
$$

Again, with $\rho=\frac{k}{2 h^{2}}$, equation (3.40) becomes

$$
\begin{aligned}
& F(r, z+1)=F(r+1, z)\left[-i \frac{\rho}{2}-i \frac{\rho h}{4 r_{s}^{\prime}}\right] \\
& -F(r-1,2)\left[-i \frac{\rho}{2}+i \frac{\rho h}{4 r_{s}^{\prime}}\right] \\
& +F(r, z)\left[1+i \rho-\frac{i \alpha k}{2(1-\alpha)}+\frac{i \alpha k}{2(1-\alpha)} e^{-F^{2}(r, z)}\right]
\end{aligned}
$$


Figure (3.3) represents the nodes involved in solving $F(r, z+1)$ for the "off axis" element.

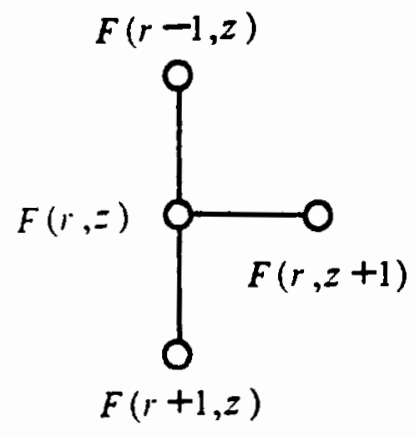

Figure 3.3. Nodal points calculation for $r>0, z=0$.

Note the similarity of figures (3.2) and (3.3). The only addition to figure (3.3) and equation (3.41) is the $F(r-1, z)$ term. This is because we can no longer assume that the adjacent nodes of $F(r, z)(F(r+1, z)$ and $F(r-1, z))$ are the same.

Up to this point we have used the forward method, the derivative with respect to the $z$ term, in determining the longitudinal elements, and the central difference method to determine the transverse element. We are unable to improve the above scheme greatly since we are at the interface, or rather, close to the boundary of our hypothetical cylinder. However, we could perhaps increase the stability of this scheme and hence its accuracy, if we were able to incorporate more nodes into our calculation of $F(r, z+1)$. Examining the above difference equation, we could change the forward difference method to a central difference method, as shown in figure (3.4), such that we use three nodes in calculating $F(r, z+1)$ in the "on axis" calculation, and four nodes for the "off axis" calculation. This method is known as the Richardson approximation. ${ }^{1,11}$ It is important to note that this scheme is unconditionally unstable when the coefficients are real. 
However, because $\mathrm{F}$ is complex, this scheme is stable within a certain region, as we shall show later.

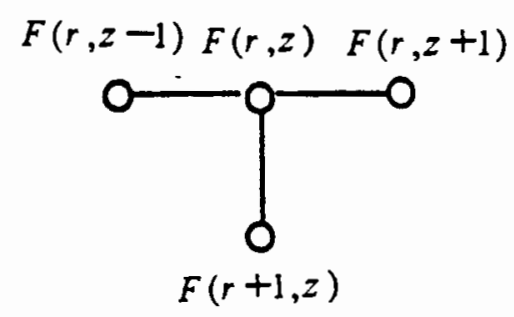

Figure 3.4. Nodal points calculation for $r=0, z>0$.

Rewriting the "on axis" equation

$$
\begin{aligned}
& \frac{F(r, z+1)-F(r, z-1)}{2 k}=-\frac{i}{2}\left[\frac{F(r+1, z)-2 F(r, z)+F(r-1, z)}{h^{2}}\right] \\
& -i \frac{\alpha}{2(1-\alpha)}\left[1-e^{-F^{2}(r, z)}\right] F(r, z)
\end{aligned}
$$

and in terms of $F(r, z+1)$

$$
\begin{aligned}
& F(r, z+1)=-\frac{i k}{h^{2}}[F(r+1, z)+F(r-1, z)] \\
& +F(r, 2)\left[\frac{i 2 k}{h^{2}}-i \frac{\alpha k}{(1-\alpha)}+i \frac{\alpha k}{(1-\alpha)} e^{-F^{\prime}(r, s)}\right]+F(r, z-1)
\end{aligned}
$$

To obtain the "off axis" model, we have

$$
\begin{aligned}
& \frac{F(r, z+1)-F(r, z-1)}{2 k}=-\frac{i}{4}\left[\frac{F(r+1, z)-2 F(r, z)+F(r-1, z)}{h^{2}}\right] \\
& -\frac{i}{4 r_{s}}\left[\frac{F(r+1, z)-F(r-1, z)}{2 h}\right]-\frac{i \alpha}{2(1-\alpha)}\left[1-e^{-r^{2}(r, z)}\right] F(r, z)
\end{aligned}
$$


If we let $\rho=\frac{k}{2 h^{2}}$, and group terms, (3.44) becomes

$$
\begin{aligned}
& F(r, z+1)=F(r+1, z)\left[-i \rho-i \frac{\rho h}{2 r_{s}}\right]+F(r-1, z)\left[-i \rho+i \frac{\rho h}{2 r_{z}}\right] \\
& +F(r, z)\left[i 2 \rho-i \frac{k \alpha}{(1-\alpha)}+i \frac{k \alpha}{(1-\alpha)} e^{-F^{2}(r, z)}\right]+F(r, z-1)
\end{aligned}
$$

The "off axis" now uses the figure (3.5), five point scheme.



Figure 3.5. Nodal points calculation for $\mathrm{r}>0, \mathrm{z}>0$.

where four nodes are actually used to determine the fifth node.

Although the equations above possess the same order of error, it has been found that greater stability is obtained if we use the second equation, (3.45), immediately after we calculate the first column of nodes.

The word "stability" has been used throughout this discussion. So what is the criterion that we must establish in order for our calculation to be deemed stable? We have seen, from equation (2.39), that the system is stable within $0<$ $\rho \leq 1 / 4$ for the cylindrical coordinate. But Lapidus and Pinder ${ }^{1}$ stated that a partial differential equation of the form

$$
U_{x}=i \omega U_{y}
$$


where $i=\sqrt{-1}, \omega=$ some real number, cannot be solved using the classical approach. In other words, the classic explicit method would yield a completely unstable approximation. However, the Richardson approach would yield a conditionally stable approximation, provided that the following is satisfied:

$$
0<\rho \leq \frac{1}{4 \omega}
$$

Recalling equation (3.31)

$$
\frac{\partial^{2} F}{\partial r^{\prime 2}}+\frac{1}{r^{\prime}} \frac{\partial F}{\partial r^{\prime}}-i 4 \frac{\partial F}{\partial z^{\prime}}+\frac{2 \alpha}{1-\alpha}\left[1-e^{-F^{2}}\right] F=0
$$

we can rewrite it as

$$
F_{z}=i \omega\left[F_{r}+\frac{1}{r} F_{r}+\frac{2 \alpha}{(1-\alpha)}\left[1-e^{-F^{2}}\right] F\right]
$$

where $i=\sqrt{-1}$ and $\omega=1 / 4$. Inserting $\omega$ into the condition, we find that Richardson's approximation is stable in the region of $0<\rho \leq 1$. This $\rho$ is larger than the $\rho$ calculated in section 2.7, so we will choose the smaller of the two; that is, for stability we will choose a value for $\rho$ that falls in the region $0<\rho \leq 1 / 4$.

We will now summarize the four equations that we derived earlier. For $\mathrm{r}=$ 0 and $z=0$, equation (3.38), $F(r, z+1)$ is

$$
-i 2 \rho F(r+1, z)+F(r, 2)\left[1+i 2 \rho-\frac{i k \alpha}{2(1-\alpha)}+\frac{i k \alpha}{2(1-\alpha)} e^{-F^{2}(r, z)}\right]
$$

When $r>0$ and $z=0$, equation (3.41), we have

$$
\begin{array}{r}
F(r+1, z)\left[-i \frac{\rho}{2}-i \frac{\rho h}{4 r_{s}^{\prime}}\right]-F(r-1, z)\left[-i \frac{\rho}{2}+i \frac{\rho h}{4 r_{s}^{\prime}}\right] \\
+F(r, z)\left[1+i \rho-\frac{i \alpha k}{2(1-\alpha)}+\frac{i \alpha k}{2(1-\alpha)} e^{-f^{2}(r, z)}\right]
\end{array}
$$


As we start to propagate away from the interface, we will switch to the central difference scheme (Richardson method). For $r=0$ and $z>0$, equation (3.43), we will use the following equation to calculate $F(r, z+1)$

$$
\begin{aligned}
& -i 2 \rho[F(r+1, z)+F(r-1, z)]+F(r, z-1) \\
& +F(r, z)\left[i 4 \rho-i \frac{\alpha k}{(1-\alpha)}+i \frac{\alpha k}{(1-\alpha)} e^{-F^{2}(r, z)}\right]
\end{aligned}
$$

The "off axis" equation for $r>0$ and $z>0$, equation (3.45), is

$$
\begin{aligned}
& F(r+1, z)\left[-i \rho-i \frac{\rho h}{2 r_{s}^{\prime}}\right]+F(r-1, z)\left[-i \rho+i \frac{\rho h}{2 r_{s}^{\prime}}\right] \\
& +F(r, z)\left[i 2 \rho-i \frac{k \alpha}{(1-\alpha)}+i \frac{k \alpha}{(1-\alpha)} e^{-F^{2}(r, z)}\right]+F(r, z-1)
\end{aligned}
$$

where $\rho=\frac{k}{2 h^{2}}$. 


\section{CHAPTER IV}

\section{DISCUSSION}

In order to construct a working model, we assumed that the beam propagates within a cylindrical cylinder filled with plasma gas. We now further stipulate that the input beam is of Gaussian form, such that

$$
E(r, 0)=E_{o} e^{-\frac{r^{2}}{w_{o}^{2}}}
$$

As before, $w_{s}$ is the minimum spot size of the beam at the interface of the cylinder, as shown in figure (4.1).

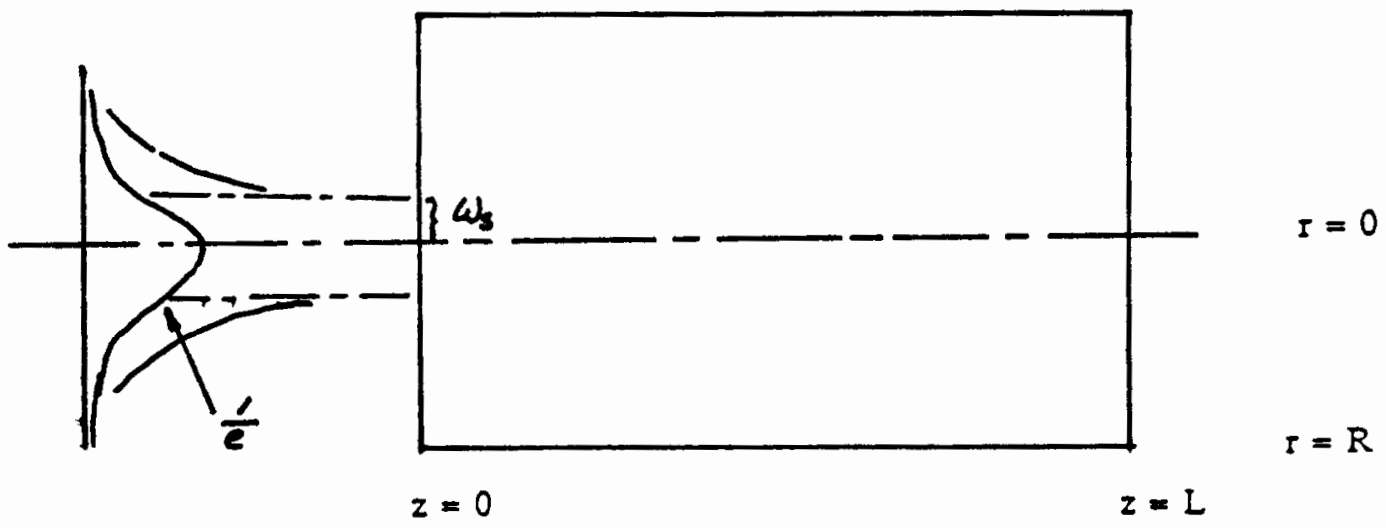

Figure 4.1. Cross-section of Gaussian beam at the interface of the plasma cylinder.

where $R$ is the radius of the cylinder, and $L$ is the length of the cylinder. 
The initial conditions are

$$
E(0,0)=E_{o}
$$

and

$$
\lim _{r \rightarrow \infty} E(r, 0)=0
$$

with the following boundary conditions

$$
E(R, z)=0
$$

Again, $R$ is the radius of the cylinder, and

$$
\frac{\partial E}{\partial r}=0 \quad \text { at } r=0
$$

We can easily prove equation (4.3b) by differentiating (4.1) with respect to $r$. Equation (4.2b) stipulates that the field, hence the intensity, must vanish to zero as $\mathrm{r}$ goes to infinity. However, for the purposes of our study, we require that field $E$ vanish to zero before it arrives at the boundary of the cylinder. So care must be taken in choosing the spot size, and the radius of the cylinder.

A matrix of 100 by 1000 was selected in obtaining the solution of the beam equation. Since there is no azimuthal variation, we can reduce a three dimensional problem to two dimensions; the field is now only a function of $r$ and $z$, by neglecting the $\phi$ dependence. If we map the matrix onto the cylinder in question, as shown in figure (4.2), 


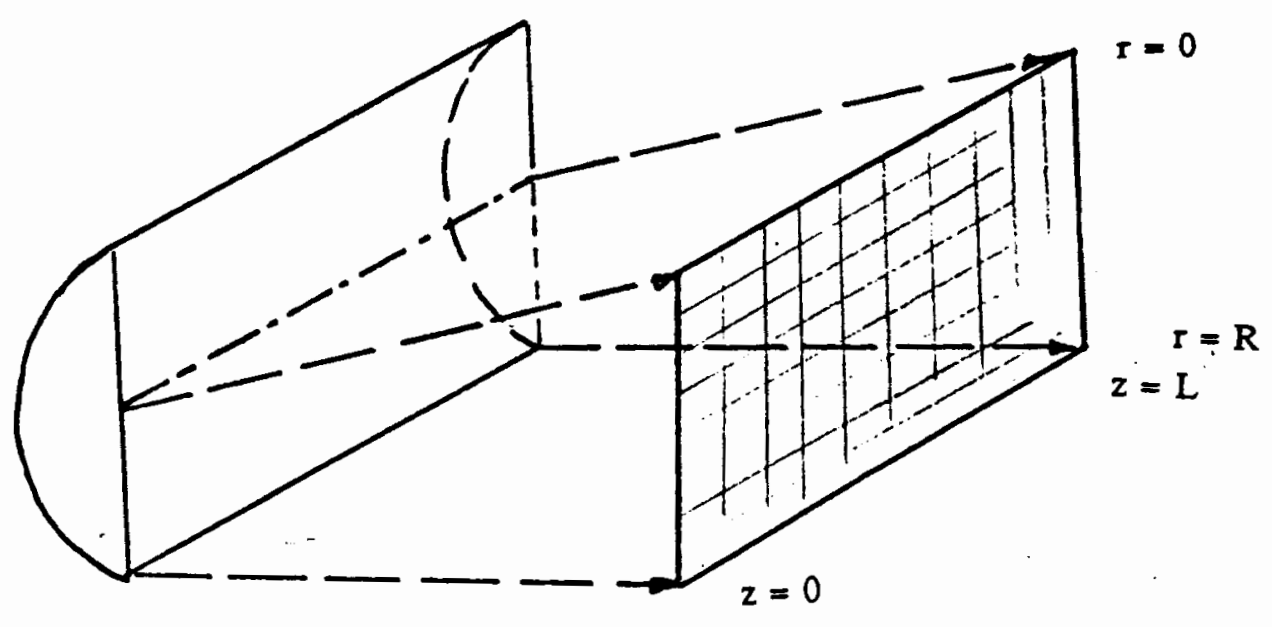

Figure 4.2. Matrix mapping on the plasma cylinder.

we can calculate the field as it propagates along the cylinder. We will only consider half of the cylinder, since we have already assumed that there is azimuthal symmetry, $\phi$. This further suggests that we could obtain the same solution if we were to flip the matrix onto its top. We will also denote the topmost row as $r=0$, and the far left column as $z=0$. The values for the initial condition are then inserted into the $\mathrm{z}=0$ column.

To examine the validity of the numerical scheme developed, the known linear case was used for the initial test. From our understanding of a Gaussian beam propagating in a vacuum, the beam must remain Gaussian with decreasing amplitude, as the beam propagates away from the origin. From the definition of Raleigh length,

$$
Z_{R}=\frac{\pi w_{s}{ }^{2}}{\lambda}
$$

where $Z_{R}$ is the Raleigh length, $w_{f}$ is the spot size, and $\lambda$ as the wavelength. 
To be consistent, we have chosen $\lambda$ to be $10.6 \mu \mathrm{m}$, the wavelength for a $\mathrm{CO}_{2}$ laser, and a normalized spot size of 100 . We also choose the beam parameter $y$ to be $3.47 * 10^{-19}$ to be consistent with Casperson and Yeung. ${ }^{6}$ Inserting these values into equation (4.4), we found the normalized Raleigh wavelength $Z_{R}$ to be 2.96 * $10^{9}$. Due to the large value of $Z_{R}$, no plot was made. However, figure (4.3) shows the beam profile, in terms of its intensity versus longitudinal and radial distance.

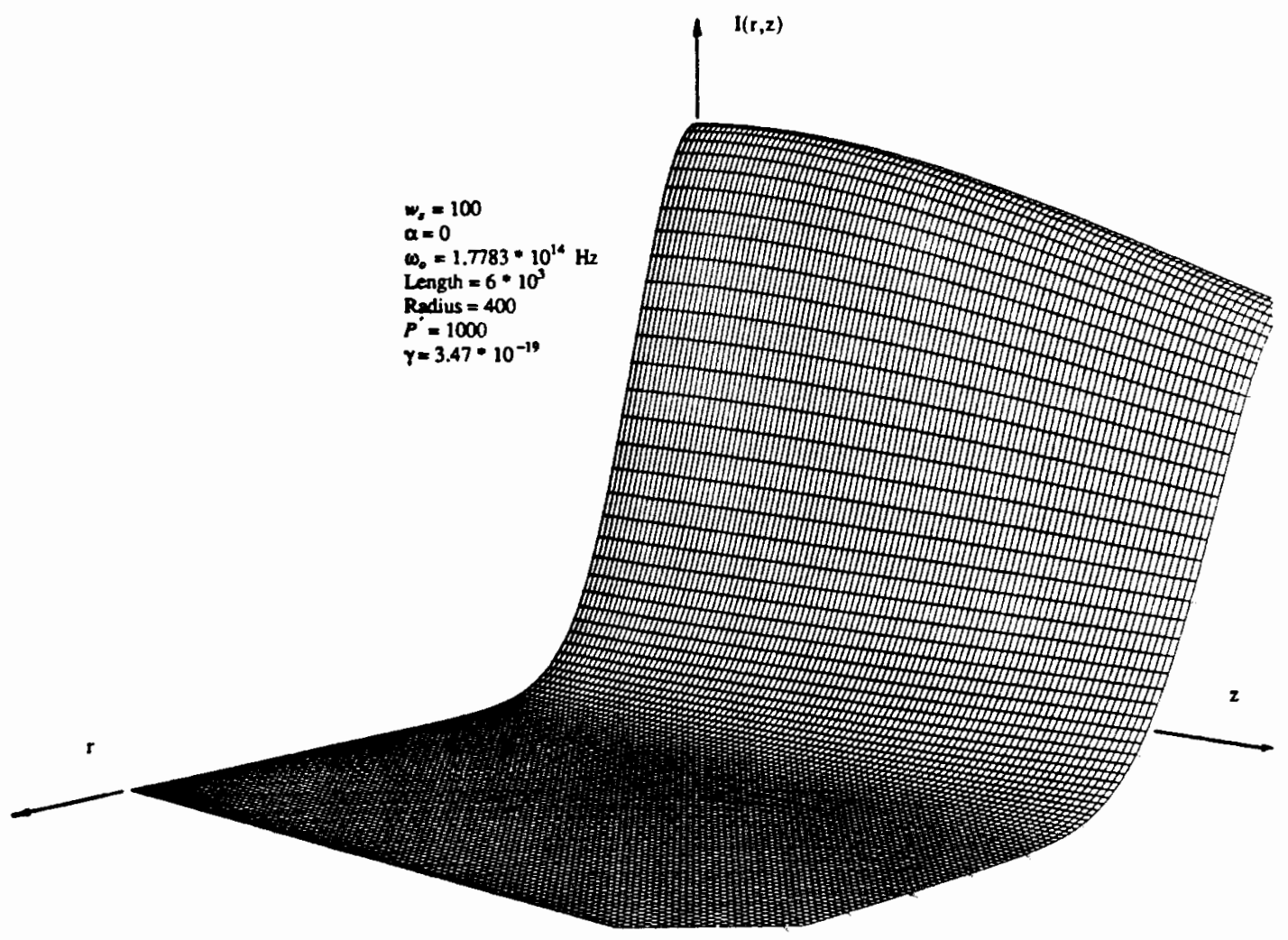

Figure 4.3. Beam intensity profile in vacuum.

Knowledge of $Z_{R}$ allows us to determine the distance required for the beam to drop its intensity to $1 / 2$ of its initial value. Inspection of figure (4.3) shows that the beam spreads out radially with increasing $z$, as predicted. Thus we conclude that the numerical solution is valid for the linear case. 
As a reminder to the reader, figures (4.4) to (4.6) are plotted with beam intensity versus (longitudinal and radial) distance. In order to capture the maximum intensity of the beam, we have scaled the plot so that the maximum intensity has the value of one on the plotter. Intensity plots of the nonlinear case, figure (4.4) to (4.6), has been plotted against the logarithmic scale. This is why the beam profile appears to be discontinuous along the radial direction.

Once the plot of the linear case shows consistency with the predicted value, we move on to the nonlinear case. Again, with $\lambda=10.6 \mu \mathrm{m}, w_{t}=100$, and normalized power $P^{\prime}=1000$. A plot of the result is shown in figure (4.4).



Figure 4.4. Beam intensity profile in plasma.

The plot shows the intensity of the beam growing exponentially with increasing $\mathrm{z}$ to a first local maximum. Beyond that point, the beam diffracts, and the beam spot size grows in proportion to the decrease in intensity.

The reason that the beam intensity peaks out, and subsequently diffracts, is due to saturation of the index of refraction. This is our first observation of "self- 
focusing". The intense radiation pressure produced by the laser causes the local index of refraction to change. Since the beam is most intense in the center, the refraction index at the center of the beam is highest. Thus, a ray near the axis experiences a net focusing force, while the periphery of the beam experiences a net diffracting force. ${ }^{8}$ This also shows that the radial intensity profile "peaks out" along the central axis of the beam and not radially outward.

As the beam continues to diffract to a local maximum spot size, the "selffocusing" force takes over again, and the process repeats itself. However, the spot size never returns to its previous minimum. This may be interpreted intuitively as showing that the beam is attempting to damp itself out, and move into a steady-state. Figure (4.5a) shows that the beam intensity eventually reaches steady-state oscillations. Figure (4.5b) is identical to that of figure (4.5a), except for slight rotation about one of its axes.

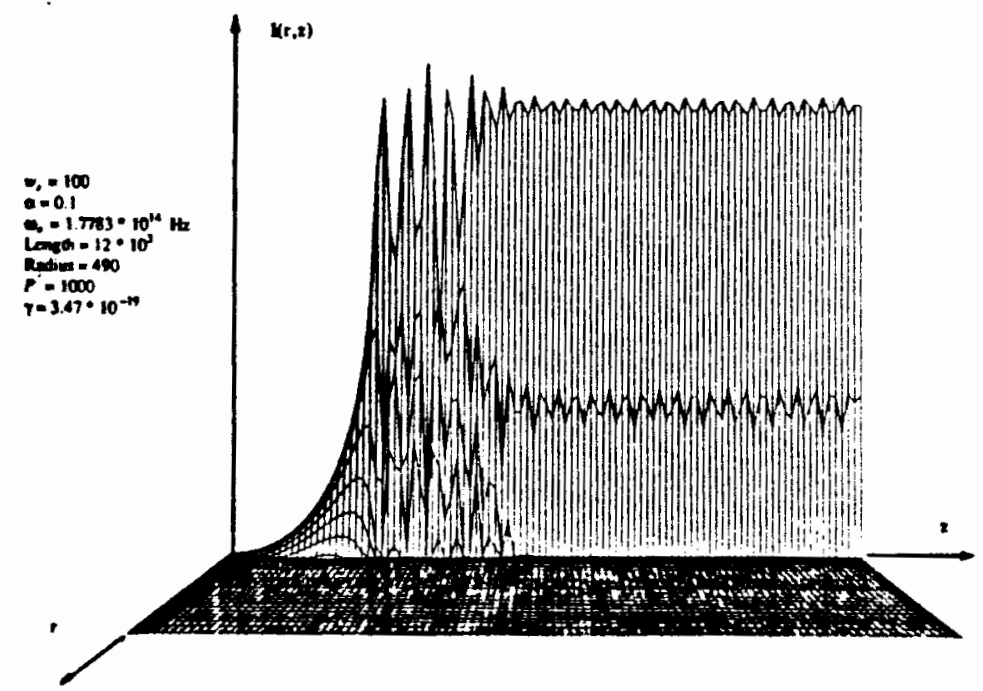

Figure 4.5(a) Steady state oscillations. 


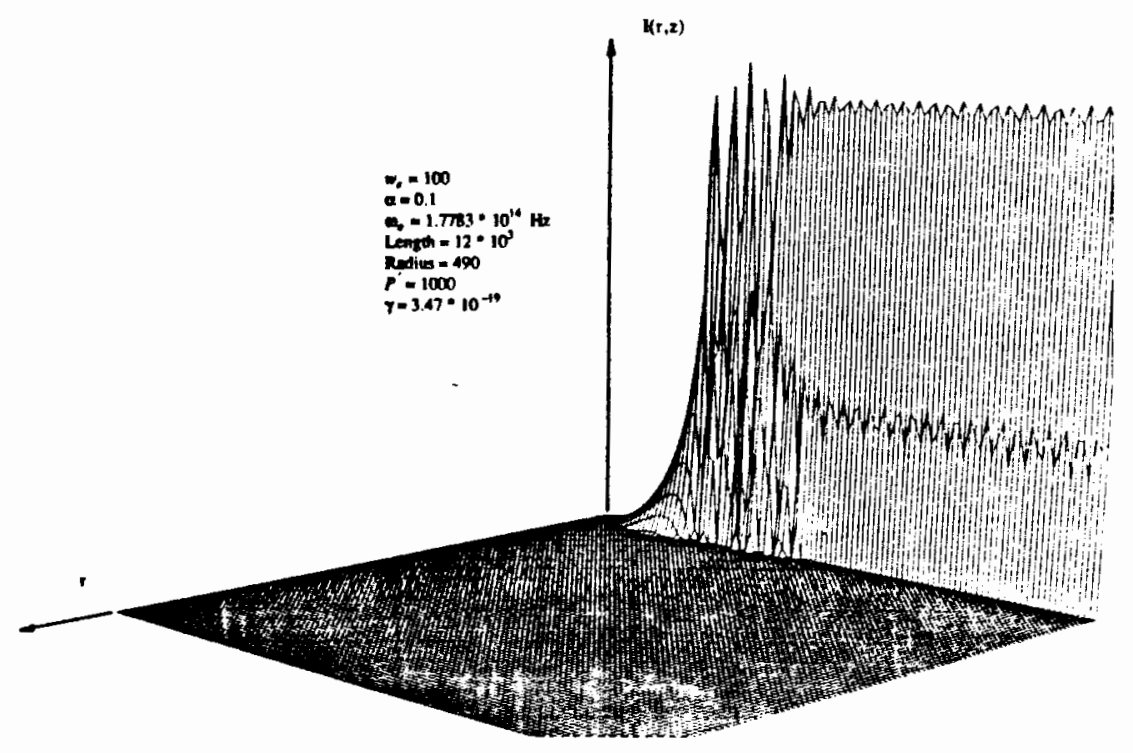

Figure 4.5(b) Steady state oscillations with rotation on intensity axis.

In the steady state region, figure (4.5a), the intensity of the beam appears to be "pinched", and hence also the spot size, as it propagates longitudinally. This is because the refractive index near the axis rises at first, but then becomes constant upon saturation. The resultant induced "convex lens" is flat in the center, therefore tends to focus incoming rays into a ring. The rays initially bent toward the axis continued inward and gives rise to a central maximum. The intensity in the right also gives rises until a new "flat" region is formed in the induced lens, where upon a new ring begins to form. 


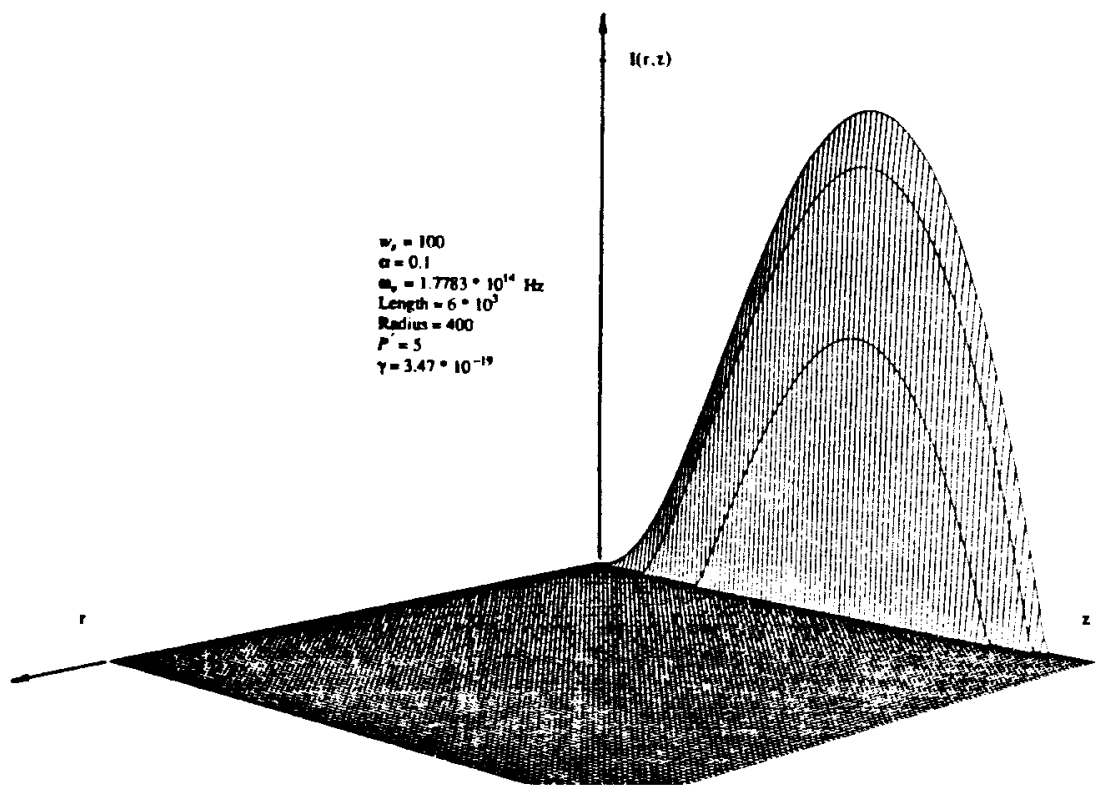

Figure 4.6. Weak focusing.

At a low input power, as shown in figure (4.6), the solution shows the "on axis" intensity gives rise initially to a "weak" focus and then falls to zero as if the beam were diffracting. 


\section{CHAPTER V}

\section{CONCLUSION}

Self-focusing phenomena of $\mathrm{CW}$ beams propagating in nonlinear media have been studied using numerical techniques. The results show that the beam intensity causes a change in the refractive index of the medium, and as a consequence, the beam intensity grows to a higher value than that of the input. Furthermore, the results show that, in a plasma, the beam never focuses down to a point because of refractive saturation. For small values of power $P^{\prime}$, a weak selffocus is observed, and beyond the local maximum intensity, the beam diverges without limit. For large values of $P^{\prime}$, there are periodic oscillations of the intensity, hence the spot size, about a steady state value. Perhaps the most important finding is the deformation of the beam profile, since it had been assumed previously that the beam profile remains constant. This assumption has been found to be only valid for small values of $P^{\prime}$.

In this paper, we have concentrated primarily on the previous work done by Casperson and Yeung. ${ }^{6}$ The major difference here is the technique utilized in determining the wave propagation within the plasma gas. Due to the success of the numerical method used in the paper, we believe that this method could be useful when investigating other topics entailing nonlinear media, and areas that require integrating nonlinear partial differential equations. The applications of self-focusing phenomena are almost endless. One of the most debated issues is 
the feasibility of a satellite-based high power laser as proposed in the Strategic Defense Initiative (SDI). One must determine beam behavior on entry to the ionosphere, at the edge of the earth's atmosphere. However, the ionosphere is a plasma gas - the topic of our discussion. During the course of our discussion we have seen that at high input powers, the beam tends to self-focus and reach a steady state. As a consequence of self-focusing, the beam intensity. This suggests applications in which a lower power laser could be used in place of the high power laser. In such applications, one could use the plasma gas itself as a focusing element. 


\section{BIBLIOGRAPHY}

1. Leon Lapidus, George F. Pinder.

Numerical Solution of Partial Differential Equations in Science and Engineering.

New York: John Wiley and Sons, 1982.

2. E. Hecht, A. Zajac.

Optics. 4th Ed. New York: Addison-Wesley Publishing, 1979.

3. William E. Boyce, Richard C. DiPrima.

Elementary Differential Equations and Boundary Value Problems. 3rd Ed.

New York: John Wiley and Sons, 1977.

4. David J. Griffiths.

Introduction to Electrodynamics. New Jersey: Prentice-Hall, Inc., 1981.

5. Ammon Yariv, Pochi Yeh.

The Application of Gaussian Beam.

Formalism to Optical Propagation in Nonlinear Media. Optical Communications. Vol 27, no. 2. Nov 1978.

6. Lee W. Casperson, Wing F. Yeung.

Self Focusing of Laser Beams in Plasma. University of California, Los Angeles, California. Thesis dissertation.

7. J. M. Sanz-Serna.

Conservative and Nonconservative Schemes for the Solution of the Nonlinear

Schrodinger Equation. IMA Journal of Numerical Analysis. Vol. 6, no. 1. 
Jan 1986.

8. E. L. Dawes, J. H. Marburger

Computer Studies in Self Focusing. Physical Review. Vol. 179, no. 3. Mar 1969.

9. M. S. Sodha, A. K. Ghatak, V. K. Tripathi.

Self Focusing of Laser Beams in Plasmas and Semiconductors.

E. Wolf, Progress in Optics XIII, 1976.

10. J. D. Jackson

Classical Electrodynamics.

New York: John Wiley and Sons, Inc. 1962.

11. W. E. Milne

Numerical Solution of Differential Equations. New York: Dover Publications, Inc. 1970.

12. W.F. AMES

Mathematics in Science and Engineering. Academic Press, Inc. 1965.

13. W. Kapilan

Advanced Mathematics for Engineers. Addison-Wesley Publishing, Co. 1981 
APPENDICES 


\section{APPENDIX I}

\section{MAIN PROGRAM}

1 \#include <stdio.h>

2 \#include <math.h>

3 \#include "def.h"

4

5 extern double Bound_Cond_wrt_R();

6 extern double $\mathrm{CEXP}($ ),CMULT(),CDIV(),CADD(),CDIV();

7 extern double R_soln, I_soln;

8 extern double intensity;

9

10 main()

11\{

12 float norm_ro, norm_z0, norm_r1, norm_z1 ;

13

14 double $\mathrm{PI}=3.1415926535897932384626433$;

15 int step_z, step_r ;

16 double length, radius, wp, spot, r_spot, mu, eps;

17 double rinc, $\mathrm{r0}, 1,10$, gamma;

18 double tmp, tmp1, tmp2, tmp_r, tmp_c, lambda ;

19 double power; 
20 double tmp3, tmp4, cons1_r, cons1_c, r_step ;

21 double a1, a2, a3, Radius ;

22 double npower, tpower;

23 double max_int ;

24 int $\mathrm{i}, \mathrm{r}, \mathrm{j}, \mathrm{z}$;

25 int z_start, z_end ;

26 double $\mathrm{Ne}, \mathrm{e}, \mathrm{m}$, opticalf;

27 double tmp_i0, tmp_i, tho ;

28 double cell_ $\mathrm{r}=100.0$;

29 double cell_z=1000.0;

30 double alpha, field ;

31

32

33 double $\mathrm{R}$, tmp0, Re,Im,kap,spotsize, dr, dz;

34

35 eps $=8.854188 \mathrm{e}-12$;

$36 \mathrm{mu}=4.0 \mathrm{e}-7 * \mathrm{PI}$;

37

38 do \{

39 printf("Please Enter Normalized Length of Cylinder: ");

40 scanf ("\%lf", \&length);

41 printf("0) ;

42 printf("Please Enter Normalized Radius of Cylinder: ");

43 scanf ("\%lf",\&radius);

44 printf("0) ;

$45 \mathrm{r} 0=$ radius/cell_ $\mathrm{r}$; 


$$
10=\text { length } / \text { cell_z; }
$$

$$
\text { rho }=10 /\left(2.0 * \mathrm{r}^{0} *_{\mathrm{r}} \mathrm{O}\right) \text {; }
$$

$48 \quad\}$

$49 \quad$ while ( rho $>0.25$ );

50

51

printf("Please Enter value for Normalized Spot Size spot: ");

52 scanf ("\%lf",\&spot);

53 printf("0);

54 printf("Please Enter the Optical Freq: (Hz*E14) ");

55 scanf("\%lf",\&opticalf) ;

$56 \quad$ opticalf $=$ opticalf $* 1.0 \mathrm{e} 14$;

57 printf("0);

58 printf("Enter Alpha: ") ;

59 scanf("\%lf",\&alpha) ;

60 printf("0) ;

$61 \mathrm{tmp}=$ opticalf*opticalf*alpha ;

$62 \mathrm{wp}=\operatorname{sqrt}(\mathrm{tmp})$;



64 printf("Please Enter Normalized Power: ") ;

65 scanf("\%lf",\&power) ;

66 printf("0);

67 printf("Please Enter value for Gamma: ");

68 scanf ("\%lf",\&gamma);

69 printf("0);

$70 \quad$ gamma $=$ gamma $* 1.0 \mathrm{e}-19 ;$ 


$$
\text { ** } \lambda=\frac{2 \pi}{w_{0} \mu \varepsilon \sqrt{1-\frac{w^{2}}{w_{0}^{2}}}}
$$

**

$$
\text { printf("Lambda }=\% \text { g0,lambda) ; }
$$


$95 \quad$ \{

96

for $(j=0 ; j<=($ int $)($ cell_z -1.0$) ; j++)$

97

98

g.x $[\mathrm{i}][\mathrm{j}]=0.0$;

99

$$
\text { g.y }[\mathrm{i}][\mathrm{j}]=0.0 \text {; }
$$

100

$101\}$

102

103 I_spot $=($ spot*lambda $) /(\mathrm{PI} * \operatorname{sqrt}(2.0))$;


*opticalf*mu*eps*(1.0-alpha)) ;

105 field $=\operatorname{sqrt}(\mathrm{tmp})$;

106 Bound_Cond_wr_R(field,spot,r0,gamma);

107

108 for $(z=0 ; z<=$ (int) (cell_z-1.0); $z++)$

109

110

$$
\text { for }(r=0 ; r<=(\text { int })(\text { cell_ } r-1.0) ; r++)
$$

111

112

f***********

113 ** Begin Numerical method

114

115

if $(r==0)$

116

117

$$
\text { if }(z<1)
$$

118 
tmpl $=\exp (-\mathrm{tmp})$;

tmp_r $=1.0$; tmp_c $=-($ alpha $* 10) /(2.0 *(1.0-$ alpha $))$; tmp_c $+=$ tmp2 ; tmp_c $+=2.0 *$ rho ; g.x $[r][z+1]=$ R_soln ; g.y $[r][z+1]=I \_s o l n$;

cons1_r $=0.0$;

cons1_c $=-2.0^{*}$ rho ; 

tmp_c $=-($ alpha*10)/(1.0-alpha $)$; tmp_c $+=\operatorname{tmp} 2$; tmp_c $+=4.0 *^{*}$ ho ; CMULT(g.x[r][z],g.y[r][z],tmp_r,tmp_c) ; g.x $[r][z+1]=R \_s o l n+g \cdot x[r][z-1]$; $\mathrm{g} \cdot \mathrm{y}[\mathrm{r}][z+1]=\mathrm{I} \_\operatorname{soln}+\mathrm{g} \cdot \mathrm{y}[\mathrm{r}][\mathrm{z}-1]$;

162

cons1_ $\mathrm{r}=0.0$;

164 cons1_c $=-4.0 *$ rho ;

165 

$\operatorname{tmp}=g \cdot x[r][z+1]^{*} g \cdot x[r][z+1]+g \cdot y[r][z+1]^{*} g \cdot y[r][z+1] ;$ tmp1 $=-0.5^{*}$ gamma ${ }^{*}$ tmp ; $\operatorname{tmp} 2=\exp (\operatorname{tmp} 1)$ tmp0 $=2.9979246 \mathrm{e} 8 * \mathrm{eps} / 2.0$; $\operatorname{tmp} 1=\operatorname{tmp} 0^{*} \mathrm{~g} \cdot \mathrm{x}[\mathrm{r}][\mathrm{z}+1]^{*} \mathrm{~g} \cdot \mathrm{x}[\mathrm{r}][\mathrm{z}+1]$; tmp2 $=\operatorname{tmp} 0 * g \cdot y[r][z+1]^{*} g \cdot y[r][z+1] ;$ $\operatorname{tmp}=\operatorname{tmp} 1+\operatorname{tmp} 2$; tmp = tmp/intensity ;

182 $\mathrm{I}[\mathrm{r}][\mathrm{z}+1]=\mathrm{tmp}$;

183

$$
\text { tmp_r }=1.0 \text {; }
$$$$
\text { tmp }=(\text { alpha } * 10) /(2.0 *(1.0 \text {-alpha })) ;
$$$$
\text { tmp0 }=g \cdot x[r][z]^{*} g \cdot x[r][z]+g \cdot y[r][z]^{*} g \cdot y[r][z] ;
$$$$
\text { tmp_c }=-\operatorname{tmp}+\mathrm{tmp}^{*}(\exp (-\mathrm{tmp} 0))+\text { tho ; }
$$
CMULT(g.x[r][z],g.y[r][z],tmp_r,tmp_c) ; g.x $[r][z+1]=R \_s o l n$; 
tmp_c $=-0.5 *$ rho $-\mathrm{rho}{ }^{*} \mathrm{r} 0 /(4.0 * \operatorname{tmp})$;

199

200

CMULT(g.x $[\mathrm{r}+1][z], g \cdot y[r+1][z]$, tmp_r,tmp_c) ;

201

g.x $[r][z+1]+=R \_s o l n$;

202

g.y $[r][z+1]+=I \_s o l n$;

203

204

tmp_r $=0.0$;

205

tmp_c $=-0.5 *$ rho + rho* $0 /(4.0 *$ tmp $)$;

206

CMULT(g.x $\left.[r-1][z], g \cdot y[r-1][z], t m p \_r, t m p \_c\right)$;

207 g.x $[r][z+1]+=R \_s o l n$;

208

g.y $[r][z+1]+=I \_s o l n$;

209

\}

210

else

211

212

tmp_r $=0.0$;

213

tmp $=($ alpha $* 10) /(1.0$-alpha $) ;$

214

tmp0 $=g \cdot x[r][z]^{*} g \cdot x[r][z]+g \cdot y[r][z]^{*} g \cdot y[r][z] ;$

215

$\operatorname{tmp} c c=-t m p+t m p *(\exp (-\operatorname{tmp} 0))+2.0 *$ rho ;

216

CMULT(g.x[r][z],g.y[r][z],tmp_r,tmp_c) ;

217

$g \cdot x[r][z+1]=R \_s o l n+g \cdot x[r][z-1]$;

218

g.y $[r][z+1]=I \_s o l n+g . y[r][z-1]$;

219

220

tmp $=($ double $) \mathrm{r} * \mathrm{rO}$;

221

tmp_r $=0.0$;

222

tmp_c $=-$ rho $-r h o * r 0 /(2.0 * \operatorname{tmp})$; 
CMULT(g.x $\left.[r+1][z], g \cdot y[r+1][z], t m p \_r, t m p \_c\right)$; g.x $[x][z+1]+=R_{-}$soln ; g.y $[r][z+1]+=I \_s o l n$;

227

228

tmp_r $=0.0$;

229 tmp_c $=-$ tho + tho*TO/(2.0*tmp);

230 CMULT(g.x[r-1][z],g.y[r-1][z],tmp_r,tmp_c) ; g.x $[\tau][z+1]+=$ R_soln ;

232 g.y $[r][z+1]+=$ I_soin ;

233 $\left.\operatorname{tmp}=g \cdot x[r][z+1]^{*} g \cdot x[r][z+1]+g \cdot y[r][z+1]^{*} g \cdot y\right]$ tmpl $=-0.5 *$ gamma*tmp ; tmp2 $=\exp (\mathrm{tmp} 1)$; tmp0 $=2.9979246 \mathrm{e} 8 * \mathrm{eps} /(2.0 * \operatorname{tmp} 2)$; tmpl $=\operatorname{tmp} 0^{*} \mathrm{~g} \cdot \mathrm{x}[\mathrm{r}][z+1]^{*} \mathrm{~g} \cdot \mathrm{x}[\mathrm{r}][\mathrm{z}+1]$; 
252 max_int $=0.0$;

253 step_z $=100$;

254 step_r $=10$;

255 for $(z=0 ; z<=$ (int) (cell_z -1.0$) ; z+=$ step_z $)$

256 \{

257 for $(r=0 ; r<=($ int $)($ cell_ $r-1.0) ; r+=$ step_ $r)$

258\{

259 if $\left(\max \_\right.$int $\left.<\mathrm{I}[\mathrm{r}][\mathrm{z}]\right)$

260 max_int $=\mathrm{I}[\mathrm{r}][\mathrm{z}]$;

$261\}$

$262\}$

263 printf("The max_int $=\%$ g0, max_int ) ;

264

265 for $(z=0 ; z<=($ int $)($ cell_z -2.0$) ; z+=$ step_z $)$

266\{

267 for $(r=0 ; r<=($ int $)($ cell_ $r-2.0) ; r+=$ step_ $r)$

$268 \quad\{$

269

270 norm_rO $=$ (float) $\mathrm{r} /$ cell_r ;

271 norm_zO = (float) $z / c e l l \_z$;

272 norm_r1 = (float) $(\mathrm{r}+$ step_r $) /$ cell_r ;

273 norm_z1 $=$ (float) $(z+$ step_z $) / c e l l \_z$;

274

275 printf("polygon 40); 
276 printf("\%f \%f \%f0,norm_ro,norm_z0,I[r][z]/max_int);

277 printf("\%f \%f \%f0,norm_rl,norm_z0,I[r+step_r][z]/max_int) ;

278 printf("\%f \%f \%fo,norm_rl,norm_z1,I[r+step_r][z+step_z]/max_int) ;

279 printf("\%f \%f \%fO,norm_ro,norm_z1,I[r][z+step_z]/max_int) ;

$280\}$

281

$282\}$

283 \} /*End of Main*/ 
APPENDIX II

\section{SUBROUTINES}

1 \#include <stdio.h>

2 \#include <math.h>

3 \#include "def.h"

4

5 double $C \exp [2]$;

6 double R_soln, I_soln ;

7 double intensity;

8

9 double

10 CEXP(re,im)

11 double re, im;

12 I

$13 \quad \exp [0]=\exp (\mathrm{re}) * \cos (\mathrm{im})$;

$14 \quad \exp [1]=\exp (\text { re })^{*} \sin (\mathrm{im})$;

15 return;

$16\}$

17

18 double

19 CMULT(a,b,c,d) 
20 double a, b, c, d ;

21 I

22 R_soln $=a^{*} c-b * d$;

23 I_soln $=b^{*} c+a * d$;

24

25 return ;

$26\}$

27

28 double

$29 \operatorname{CDIV}(\mathrm{a}, \mathrm{b}, \mathrm{c}, \mathrm{d})$

30 double $a, b, c, d$;

31\{

32

33 R_soln $=\left(a^{*} c+b * d\right) /\left(c^{*} c+d^{*} d\right)$;

34 I_soln $=\left(b^{*} c-a^{*} d\right) /\left(c * c+d^{*} d\right)$;

35

36

return ;

37 \}

38

39 double

$40 \operatorname{CADD}(a, b, c, d)$

41 double a, b, c, d;

42 l

$43 \quad$ R_soln $=a+c$;

44 I_soln $=b+d$; 
46 return ;

47 \}

48

49 double

$50 \operatorname{CSUB}(a, b, c, d)$

51 double a, b, c, d;

52\{

$53 \quad$ R_soln $=\mathrm{a}-\mathrm{c}$;

54 I_soln = b - d ;

55

56 return;

$57\}$

58

59 double

60 Bound_Cond_wrt_R(fi,spot,dr,gamma)

61 double fi, spot, dr, gamma ;

62\{

63 double $\mathrm{PI}=3.1415926535897932384626433$;

64 double step_r, field;

65 double w0, lambda;

66 double ws, wo,power,alpha ;

67 double tmp, tmp1, $\tau_{\text {_inc }}=0.0$;

68 int $\mathrm{i}, \mathrm{r}, \mathrm{z}$;

69 double eps $=8.854188 \mathrm{e}-12, \mathrm{mu}=4.0 \mathrm{e}-7 * \mathrm{PI}$;

70 double tmp2;

71 double tmp0; 
72 double count[100];

73

74 for $(r=0 ; r<=99 ; r++)$

75

76

$r_{\text {inc }}=\mathrm{dr} *($ double $) \mathrm{r}$;

77

g.x $[r][0]=f^{*} \exp \left(-r \_i n{ }^{*}{ }_{-} \_i n c /\left(\right.\right.$ spot ${ }^{*}$ spot $\left.)\right)$;

78

g. $\mathrm{y}[\mathrm{r}][0]=0.0$;

79

$$
\operatorname{tmp}=\mathrm{g} \cdot \mathrm{x}[\mathrm{r}][0]^{*} \mathrm{~g} \cdot \mathrm{x}[\mathrm{r}][0] \text {; }
$$

80 tmp1 $=-0.5 *$ gamma*tmp ;

81 tmp2 $=\exp ($ tmp 1$)$;

82 tmp0 $=2.9979246 \mathrm{e} 8 * 8.854188 \mathrm{e}-12 / 2.0$;

83 tmp $1=\operatorname{tmp} 0 * \mathrm{~g} \cdot \mathrm{x}[\mathrm{r}][0]^{*} \mathrm{~g} \cdot \mathrm{x}[\mathrm{r}][0]$;

84 if $(r=0)$

85

86 intensity $=\mathrm{tmp} 1$;

87 $\mathrm{I}[0][0]=\mathrm{tmp} 1 /$ intensity ;

88

\}

89

else

90

$\mathrm{I}[\mathrm{r}][0]=\mathrm{tmpl} /$ intensity ;

91 tmp0 $=2.0 * \mathrm{PI} * \mathrm{dr} * \mathrm{dr} * \operatorname{tmp} 1 *($ double $) \mathrm{r}$;

92 $\operatorname{count}[\mathrm{r}]=\operatorname{tmp} 0$;

93

]

94

95 return ;

$96\}$ 


\section{APPENDIX III}

\section{DEF FILES}

\section{1 struct mat}

21

3 double $x[200][1500]$;

4 double y[200][1500] ;

$5\}$

6 ;

7

8 struct mat $\mathrm{g}$;

9

10 struct Complex

11 I

12 double Q,A ;

13 double Cexp;

14 \};

15 struct Complex Re;

16 struct Complex Im;

17

18 float I[200][1500]; 